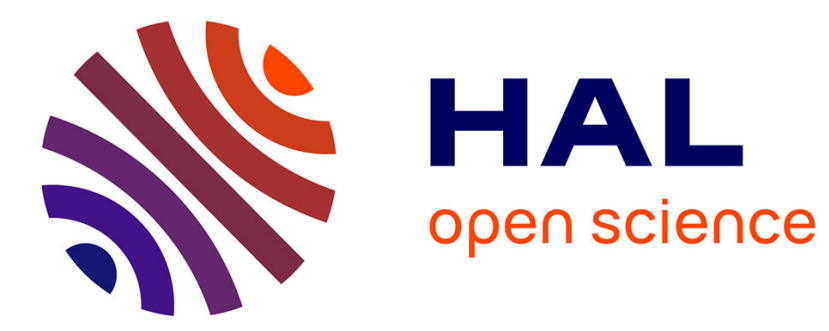

\title{
Evolutionary dynamics in discrete time for the perturbed positive definite replicator equation
}

Amie Albrecht, Konstantin Avrachenkov, Phil Howlett, Geetika Verma

\section{To cite this version:}

Amie Albrecht, Konstantin Avrachenkov, Phil Howlett, Geetika Verma. Evolutionary dynamics in discrete time for the perturbed positive definite replicator equation. The ANZIAM Journal, 2020, 62 (2), pp.148-184. hal-02892186

\section{HAL Id: hal-02892186 https://hal.inria.fr/hal-02892186}

Submitted on 7 Jul 2020

HAL is a multi-disciplinary open access archive for the deposit and dissemination of scientific research documents, whether they are published or not. The documents may come from teaching and research institutions in France or abroad, or from public or private research centers.
L'archive ouverte pluridisciplinaire $\mathbf{H A L}$, est destinée au dépôt et à la diffusion de documents scientifiques de niveau recherche, publiés ou non, émanant des établissements d'enseignement et de recherche français ou étrangers, des laboratoires publics ou privés. 


\title{
Evolutionary dynamics in discrete time for the perturbed positive definite replicator equation
}

\author{
Amie Albrecht, Konstantin Avrachenkov, Phil Howlett, Geetika Verma \\ Addresses: Amie Albrecht, Phil Howlett, Geetika Verma, \\ Scheduling and Control Group, \\ Centre of Industrial and Applied Mathematics, \\ University of South Australia. \\ Konstantin Avrachenkov, \\ INRIA. \\ Email contact: amie.albrecht@unisa.edu.au
}

July 7,2020

\begin{abstract}
The population dynamics for the replicator equation are well studied in continuous time but there is less work that explicitly considers the evolution in discrete time. The discrete-time dynamics can often be justified indirectly, by establishing the relevant evolutionary dynamics for the corresponding continuous-time system, and then appealing to an appropriate approximation property. In this paper we study the discrete-time system directly, and establish basic stability results for the evolution of a population defined by a positive definite system matrix, where the population is disrupted by random perturbations to the genotype distribution either through migration or mutation, in each successive generation.
\end{abstract}

Mathematics subject classification: 92D25, 93C10, 93C55, 93C73, 93E15 Keywords and phrases: Replicator equation, discrete-time, random perturbations

\section{Introduction}

The replicator equation was introduced in $[18,19]$ to describe the dynamical evolution over time of a population of different genotypes and to provide a systematic setting for the fundamental theorem 
of natural selection [5] which states that the rate of increase in fitness of any organism at any time is equal to its genetic variance in fitness at that time. A comprehensive review by Hofbauer and Sigmund [8] presents a detailed description of the replicator dynamics and the basic theoretical results for the standard continuous-time models with a particular emphasis on the game-theoretic interpretation. The analogous discrete-time models are reviewed much more succinctly and are essentially described by reference to the corresponding continuous-time models. The discrete-time model in [8] is presented in an archetypal form that incorporates a background payoff parameter $c$. The authors note that the dynamics of the discrete-time model "can offer more complex behaviour than the dynamics of the continuous-time model" but observe that many of the fundamental results remain valid and that the additional complexities can be "tuned down by increasing $c$ ".

Continuous-time replicator dynamics with noise or shocks has been investigated in a number of papers. The article by Foster and Young [6] was the first such article. In their original model the noise is added directly to the population frequencies. A subsequent paper by Fudenberg and Harris [7] argues that it is more natural to add noise to the population and then apply the Itô formula thus obtaining a stochastic differential equation for the population frequencies. This subsequent model does indeed appear to be more tractable than the original model and has been followed up by several other authors $[2,9,10]$. Nevertheless Khasminskii and Potsepun [11] took a different view and suggested that a Stratonovich formalism could be used to overcome a significant boundary problem in the original model. More recently Mertikopoulos and Viossat [15] add a geometric-type Brownian motion directly to the replicator dynamics while Avrachenkov and Borkar [1] first converted the standard dynamics to a gradient system on a sphere, and then added a Brownian motion on the manifold.

The replicator dynamics with noise appears to be much less investigated in discrete time than in continuous time. There are many similarities between continuous-time and discrete-time replicator dynamics. However, the replicator dynamics with noise in discrete time obtained in our formulation is very different from that obtained in existing continuous time models. As will be seen from the exposition here, the discrete-time replicator dynamics is significantly more robust to noise and perturbations than is the case in the apparently analogous continuous-time models. This can be partly explained by the fact that the Brownian motion used in the continuous-time models is an unbounded noise, whereas the natural definition of noise in the discrete-time model is inherently bounded.

We assume some familiarity with [8] but our analysis relies only on standard linear algebra and is neither directly related to, nor dependent on, other results in the existing literature. For more information we refer to Kingman [12], Mulholland and Smith [16], a more recent review by Cressman and Tao [4], and books by Sandholm [17] and Weibull [20]. A general discussion about set-valued dynamical systems can be found in McGehee [14]. 


\section{The replicator equation in discrete time}

For each $i=1, \ldots, n$ let $N_{i}$ be the number of alleles of type $\mathcal{G}_{i}$ in the population and let $N=\sum_{i=1}^{n} N_{i}$ be the total number of alleles over all types. Therefore $p_{j}=N_{j} / N$ is the proportion of allele $\mathcal{G}_{j}$ for each $j=1, \ldots, n$. For each $i \in\{1, \ldots, n\}$ the probability that a $\mathcal{G}_{i}$ allele will combine with a $\mathcal{G}_{j}$ allele in the current generation $t \in \mathbb{N}$ is $p_{j}(t)$ for each $j=1, \ldots, n$. The vector $\boldsymbol{p}(t)=\left[p_{j}(t)\right] \in \mathbb{R}^{n}$ is the relative frequency vector for the population in generation $t$. The fitness of each $\mathcal{G}_{i}$ allele is defined as $w_{i}(t)=w_{i}[\boldsymbol{p}(t)]$ where $w_{i}=w_{i}[\boldsymbol{p}]=\sum_{j=1}^{n} w_{i j} p_{j}$ for each $i=1, \ldots, n$ and $W=\left[w_{i j}\right] \in \mathbb{R}^{n \times n}$ is a constant matrix with $w_{i j} \geq 0$. The average fitness in the population is given by $w(t)=w[\boldsymbol{p}(t)]$ where $w=w[\boldsymbol{p}]=\sum_{i=1}^{n} w_{i}[\boldsymbol{p}] p_{i}=\sum_{i=1}^{n} \sum_{j=1}^{n} w_{i j} p_{i} p_{j}$. The expected total number of $\mathcal{G}_{i}$ alleles in the next generation $t+1$ is

$$
N_{i}(t+1)=w_{i}(t) N_{i}(t)
$$

for each $i=1, \ldots, n$. By summation over all $i=1, \ldots, n$ we obtain

$$
N(t+1)=\sum_{i=1}^{n} w_{i}(t) N_{i}(t)=\left[\sum_{i=1}^{n} w_{i}(t) p_{i}(t)\right] N(t)=w(t) N(t)
$$

and hence we obtain

$$
p_{i}(t+1)=N_{i}(t+1) / N(t+1)=\left[w_{i}(t) / w(t)\right]\left[N_{i}(t) / N(t)\right]=\left[w_{i}(t) / w(t)\right] p_{i}(t)
$$

for each $i=1, \ldots, n$. We can write this equation collectively in matrix form as

$$
\boldsymbol{p}(t+1)=\frac{\boldsymbol{p}(t) \circ(W \boldsymbol{p}(t))}{\boldsymbol{p}(t)^{T} W \boldsymbol{p}(t)}
$$

where we define the Hadamard product by the formula $\boldsymbol{x} \circ \boldsymbol{y}=\left[x_{i} y_{i}\right] \in \mathbb{R}^{n}$ for each $\boldsymbol{x}=\left[x_{i}\right] \in \mathbb{R}^{n}$ and $\boldsymbol{y}=\left[y_{i}\right] \in \mathbb{R}^{n}$. The collective equation (1) is known as the replicator equation in discrete time.

\section{The main results}

We consider the evolution in discrete time of a population with a finite number of distinct genotypes where the dynamics are described by a standard replicator equation [8, Section 4.3, p 503] with a system matrix that is positive definite and where each successive generation is subject to a small random perturbation of the genotype distribution either by mutation or migration. For the corresponding deterministic system with no random perturbations the fundamental theorem of natural selection ensures that, except for a finite number of special initial distributions, the population will always evolve to a pure population consisting of a single genotype. The limiting pure populations are not necessarily unique and if more than one such limit is possible then the limit will depend on the initial genotype distribution. We say that a particular genotype is conditionally dominant or locally stable if there exists an associated relative frequency threshold such that if the relative frequency of 
that genotype exceeds the corresponding threshold in any generation then the unperturbed population will necessarily converge to a pure population consisting of that genotype only. There is always at least one conditionally dominant genotype when the evolution is defined by a positive definite matrix, but there may be more than one. Indeed, with our definition, it is not unusual to find that every genotype is conditionally dominant. We note in passing that positive definite system matrices describe coordination games [3].

We begin our theoretical considerations by considering a population with only two genotypes where the replicator equation is a strictly increasing map $F_{\alpha}:[0,1] \rightarrow[0,1]$, defined by a parameter $\alpha \in$ $(0,1)$, and a single genotype proportion $p$ with an unstable fixed point $p^{*}=p_{\alpha}^{*} \in(0,1)$. In Theorem 2 we show that for sufficiently small perturbations of the population defined by a parameter $\epsilon \in(0,1)$ there are two proliferation intervals $\left[\xi_{1}(\epsilon), \xi_{0}(\epsilon)\right] \subseteq\left(0, p^{*}\right)$ and $\left[\eta_{0}(\epsilon), \eta_{1}(\epsilon)\right] \subseteq\left(p^{*}, 1\right)$ such that if the proportion enters one or other of these intervals it must forever remain within the associated capture interval $\left[0, \xi_{0}(\epsilon)\right] \supseteq\left[\xi_{1}(\epsilon), \xi_{0}(\epsilon)\right]$ or $\left[\eta_{0}(\epsilon), 1\right] \supseteq\left[\eta_{0}(\epsilon), \eta_{1}(\epsilon)\right]$. In set-mapping terminology we show that $F_{\alpha}\left((1-\epsilon)\left[0, \xi_{0}(\epsilon)\right]+\epsilon[0,1]\right) \subseteq\left[0, \xi_{0}(\epsilon)\right]$ and that $F_{\alpha}\left((1-\epsilon)\left[\eta_{0}(\epsilon), 1\right]+\epsilon[0,1]\right) \subseteq\left[\eta_{0}(\epsilon), 1\right]$. In Theorem 3 we extend this result to show that for each infinite sequence of independent, identically distributed random perturbations of sufficiently small magnitude, the proportion of each genotype over all generations will lie within the set $\left[0, \xi_{1}(\epsilon)\right] \cup\left[\eta_{1}(\epsilon), 1\right]$ with probability one. These results pave the way for similar, more general, results in higher dimensions.

Consider a conditionally dominant genotype $\mathcal{G}_{j}$ for some $j \in\{1,2, \ldots, n\}$ in a population of $n$ distinct genotypes where the replicator equation is defined by a symmetric, positive definite matrix and where there are perturbations - either random or deterministic - of sufficiently small magnitude $\epsilon \in\left(0, \epsilon_{j, \max }\right) \subset(0,1)$ in successive generations. We show that there is a closed convex proliferation set $\mathcal{D}_{j, \epsilon}=\mathcal{S}_{n-1} \cap \mathcal{E}_{j, \epsilon}$ where $\mathcal{S}_{n-1} \subset \mathbb{R}^{n}$ is the $(n-1)$-dimensional simplex and $\mathcal{E}_{j, \epsilon}$ is an $(n-1)$ dimensional ellipsoid defined in the extended $(n-1)$-dimensional hyperplane $\mathcal{H}_{n-1} \supset \mathcal{S}_{n-1}$ such that if the population distribution vector $\boldsymbol{p}$ lies in the set $\mathcal{D}_{j, \epsilon}$ at generation $s$ then the relative frequency of genotype $\mathcal{G}_{j}$ will increase in the transition to generation $s+1$ irrespective of the perturbation. This is the first part of our Theorem 4.

We extend Theorem 4 to show that for each conditionally dominant genotype $\mathcal{G}_{j}$ with a perturbation of sufficiently small magnitude $\epsilon \in\left(0, \epsilon_{j, \max }\right)$ in each generation there is an associated relative frequency threshold $\alpha_{j, \epsilon}$ such that if the relative frequency $p_{j}=\boldsymbol{e}_{j}{ }^{T} \boldsymbol{p}$ of genotype $\mathcal{G}_{j}$ at generation $s$ exceeds the threshold $\alpha_{j, \epsilon}$ then the relative frequency of genotype $\mathcal{G}_{j}$ will exceed that threshold in all subsequent generations $t>s$, irrespective of the perturbation. We will say that the associated closed convex set $\mathcal{C}_{j, \epsilon}=\left\{\boldsymbol{p} \in \mathcal{S}_{n-1} \mid p_{j} \geq \alpha_{j, \epsilon}\right\}$ is a capture set for the conditionally dominant genotype $\mathcal{G}_{j}$. This is the second part of our Theorem 4. If we denote our unperturbed map by $F: \mathcal{S}_{n-1} \rightarrow \mathcal{S}_{n-1}$ then this result asserts, in set-mapping terminology, that $(1-\epsilon) F\left(\mathcal{C}_{j, \epsilon}\right)+\epsilon \mathcal{S}_{n-1} \subseteq \mathcal{C}_{j, \epsilon}$. If the perturbations are sufficiently small, and are defined by a sequence of independent identically distributed random variables where the associated cumulative distribution function is strictly monotone, then we show that capture in some set $\mathcal{C}_{j, \epsilon}$ is inevitable. This is Theorem 5. 
Although our methodology is reliant in principle on the properties of symmetric matrices, we will show that the detailed arguments can be modified so that the main results are extended to systems where the replicator equation is defined by a matrix that is positive definite but no longer symmetric. The details are given in Section 7.

We introduce our discussion and illustrate our results with appropriate examples to highlight the underlying ideas. The important insight is that there is a special closed ellipsoidal set for each stable vertex in the unperturbed model where the relative frequency of the corresponding conditionally dominant genotype always increases in the transition to the next generation. If we restrict our attention to a subset where the increase is bounded below and if the magnitude of the subsequent random perturbation is less than the corresponding lower bound, then the relative frequency of that genotype in the perturbed model will also increase in the transition to the next generation.

\section{Notation and basic principles}

Let $W=\left[w_{i j}\right] \in \mathbb{R}^{n \times n}$ be symmetric and positive definite with $w_{i j} \geq 0$ for all $i, j=1, \ldots, n$. For each $n \in \mathbb{N}$ let $\mathcal{S}_{n-1}=\left\{\boldsymbol{p} \in \mathbb{R}^{n} \mid \boldsymbol{p}^{T} \mathbf{1}=1\right.$ and $\left.\boldsymbol{p} \geq \mathbf{0}\right\} \subset \mathbb{R}^{n}$ denote the unit $(n-1)$-dimensional simplex where $\mathbf{1}=[1] \in \mathbb{R}^{n}$ and $\mathbf{0}=[0] \in \mathbb{R}^{n}$. If $\boldsymbol{p} \in \mathcal{S}_{n-1} \subset \mathbb{R}^{n}$ denotes the genotype relative frequency distribution and we define $\boldsymbol{w}(\boldsymbol{p})=W \boldsymbol{p} \in \mathbb{R}^{n}$ and $w_{j}(\boldsymbol{p})=\boldsymbol{e}_{j}^{T} \boldsymbol{w}(\boldsymbol{p}) \in \mathbb{R}$ where $\boldsymbol{e}_{j}=\left[\delta_{i j}\right]_{i=1}^{n} \in \mathbb{R}^{n}$ is the usual Euclidean unit vector with $\delta_{j j}=1$ and $\delta_{i j}=0$ for $i \neq j$, then $w_{j}(\boldsymbol{p})$ is the fitness of genotype $\mathcal{G}_{j}$ for each $j=1, \ldots, n$ and $w(\boldsymbol{p})=\boldsymbol{p}^{T} \boldsymbol{w}(\boldsymbol{p})=\boldsymbol{p}^{T} W \boldsymbol{p} \in \mathbb{R}$ is the average fitness of the population. We also define $\hat{\boldsymbol{w}}(\boldsymbol{p})=\boldsymbol{w}(\boldsymbol{p}) / w(\boldsymbol{p})=\boldsymbol{w}(\boldsymbol{p}) / \boldsymbol{p}^{T} W \boldsymbol{p}$. Let $\boldsymbol{p}(t) \in \mathcal{S}_{n-1} \subset \mathbb{R}^{n}$ denote the distribution at time $t \in \mathbb{N}$ and assume that the distribution evolves according to the discrete-time replicator equation

$$
\boldsymbol{p}(t+1)=\boldsymbol{p}(t) \circ \boldsymbol{w}(t) / w(t)=\boldsymbol{p}(t) \circ \hat{\boldsymbol{w}}(t)
$$

for each $t \in \mathbb{N}$ where we write $\boldsymbol{w}(t)=\boldsymbol{w}[\boldsymbol{p}(t)], w(t)=w[\boldsymbol{p}(t)]$, and $\hat{\boldsymbol{w}}(t)=\hat{\boldsymbol{w}}[\boldsymbol{p}(t)]$ for convenience. We can write this equation in the alternative equivalent form

$$
\boldsymbol{p}(t+1)-\boldsymbol{p}(t)=\boldsymbol{p}(t) \circ[\hat{\boldsymbol{w}}(t)-\mathbf{1}]
$$

for each $t \in \mathbb{N}$. The alternative form (3) is useful when comparing the corresponding discrete-time and continuous-time evolutions. We have the following fundamental result. There are different versions of this result and so for the convenience of readers we include an elementary proof that is valid when $W$ is symmetric and positive definite. For earlier proofs which simply assume that $W$ is symmetric, see $[12,16]$ as well as our later discussion.

Theorem 1 Let $W=\left[w_{i j}\right] \in \mathbb{R}^{n \times n}$ be symmetric and positive definite with $w_{i j} \geq 0$ for all $i, j=$ $1, \ldots, n$. If $\boldsymbol{p}(1)>\mathbf{0}$ and $\boldsymbol{p}(1) \neq W^{-1} \mathbf{1} /\left(\mathbf{1}^{T} W^{-1} \mathbf{1}\right)$ then the average fitness of the population is a strictly increasing sequence along every uniquely defined evolutionary path with

$$
w(t+1)-w(t)=[\boldsymbol{p}(t) \circ \hat{\boldsymbol{w}}(t)]^{T} W[\boldsymbol{p}(t) \circ \hat{\boldsymbol{w}}(t)]-w(t)>0
$$


for each $t \in \mathbb{N}$. If $\boldsymbol{p}(1)>\mathbf{0}$ and $\boldsymbol{p}(1)=W^{-1} \mathbf{1} /\left(\mathbf{1}^{T} W^{-1} \mathbf{1}\right)$ then $\boldsymbol{p}(t)=\boldsymbol{p}(1)$ and $w(t)=w(1)$ for all $t \in \mathbb{N}$.

Proof Let $\Delta \boldsymbol{p}(t)=\boldsymbol{p}(t+1)-\boldsymbol{p}(t)=\boldsymbol{p}(t) \circ \hat{\boldsymbol{w}}(t)-\boldsymbol{p}(t)$. We have

$$
\begin{aligned}
\Delta \boldsymbol{p}(t)^{T} W \Delta \boldsymbol{p}(t) & =[\boldsymbol{p}(t) \circ \hat{\boldsymbol{w}}(t)-\boldsymbol{p}(t)]^{T} W[\boldsymbol{p}(t) \circ \hat{\boldsymbol{w}}(t)-\boldsymbol{p}(t)] \\
& =\{\boldsymbol{p}(t) \circ \hat{\boldsymbol{w}}(t)\}^{T} W\{\boldsymbol{p}(t) \circ \hat{\boldsymbol{w}}(t)\}-2 w(t)\|\sqrt{\boldsymbol{p}}(t) \circ \hat{\boldsymbol{w}}(t)\|^{2}+w(t) \\
& \leq\{\boldsymbol{p}(t) \circ \hat{\boldsymbol{w}}(t)\}^{T} W\{\boldsymbol{p}(t) \circ \hat{\boldsymbol{w}}(t)\}-w(t)
\end{aligned}
$$

where $\sqrt{\boldsymbol{p}}=\left[\sqrt{p_{i}}\right] \in \mathbb{R}^{n}$ and where the Cauchy-Bunyakovski-Schwarz inequality

$$
w(t)=\sqrt{\boldsymbol{p}}(t)^{T}[\sqrt{\boldsymbol{p}}(t) \circ \boldsymbol{w}(t)] \leq\|\sqrt{\boldsymbol{p}}(t)\| \cdot\|\sqrt{\boldsymbol{p}}(t) \circ \boldsymbol{w}(t)\|=\|\sqrt{\boldsymbol{p}}(t) \circ \boldsymbol{w}(t)\|
$$

has been used to deduce that $1 \leq\|\sqrt{\boldsymbol{p}}(t) \circ \hat{\boldsymbol{w}}(t)\|$. Therefore

$$
w(t+1)-w(t)=[\boldsymbol{p}(t) \circ \hat{\boldsymbol{w}}(t)]^{T} W[\boldsymbol{p}(t) \circ \hat{\boldsymbol{w}}(t)]-w(t) \geq \Delta \boldsymbol{p}(t)^{T} W \Delta \boldsymbol{p}(t) \geq 0 .
$$

It follows from (4) that for any $\boldsymbol{p} \in \mathcal{S}_{n-1}$ we have

$$
w(\boldsymbol{p})=\|\sqrt{\boldsymbol{p}} \circ \boldsymbol{w}(\boldsymbol{p})\| \Longleftrightarrow \sqrt{\boldsymbol{p}} \circ \boldsymbol{w}(\boldsymbol{p})=\alpha \sqrt{\boldsymbol{p}} \Longleftrightarrow \boldsymbol{w}(\boldsymbol{p})=\alpha \mathbf{1} \Longleftrightarrow W \boldsymbol{p}=\alpha \mathbf{1}
$$

for some $\alpha \in \mathbb{R}$ with $\alpha>0$. Since $\boldsymbol{p}=\alpha W^{-1} \mathbf{1}$ and $\boldsymbol{p}^{T} \mathbf{1}=1$ we have $\alpha=1 /\left(\mathbf{1}^{T} W^{-1} \mathbf{1}\right)$ and hence $\boldsymbol{p}=W^{-1} \mathbf{1} /\left(\mathbf{1}^{T} W^{-1} \mathbf{1}\right)$. We conclude that $w(t+1)-w(t)=0$ if and only if $\boldsymbol{p}(t)=W^{-1} \mathbf{1} /\left(\mathbf{1}^{T} W^{-1} \mathbf{1}\right)$ for all $t \in \mathbb{N}$.

Fixed points are important in population evolution. If $W^{-1} \mathbf{1}>\mathbf{0}$ then $\boldsymbol{p}^{*}=W^{-1} \mathbf{1} /\left(\mathbf{1}^{T} W^{-1} \mathbf{1}\right)>\mathbf{0}$. Thus $\boldsymbol{p}^{*} \in \mathcal{S}_{n-1}$. In this case the minimum value of $w(\boldsymbol{p})$ occurs at $\boldsymbol{p}^{*}$. Since Theorem 1 shows that $w(t+1)>w(t)$ provided $\boldsymbol{p}(t) \neq \boldsymbol{p}^{*}$ it follows that $\boldsymbol{p}^{*}$ is an unstable fixed point. Let $\mathcal{H}_{n-1}=$ $\left\{\boldsymbol{p} \in \mathbb{R}^{n} \mid \boldsymbol{p}^{T} \mathbf{1}=1\right\} \subset \mathbb{R}^{n}$ be the $(n-1)$-dimensional hyperplane containing $\mathcal{S}_{n-1}$. If we define $V_{\delta}(\boldsymbol{p})=\left\{\boldsymbol{q} \in \mathcal{H}_{n-1} \mid\|\boldsymbol{q}-\boldsymbol{p}\|<\delta\right\}$ for each $\boldsymbol{p} \in \mathcal{H}_{n-1}$ and each $\delta>0$ then the collection $\left\{V_{\delta}(\boldsymbol{p})\right\}$ over all such $\boldsymbol{p}$ and $\delta$ defines a collection of open neighbourhoods in $\mathcal{H}_{n-1}$ and hence defines an induced topology. With this topology $\mathcal{H}_{n-1}$ is isomorphic to $\mathbb{R}^{n-1}$ and we write $\mathcal{H}_{n-1} \cong \mathbb{R}^{n-1}$. The condition $W^{-1} \mathbf{1}>\mathbf{0}$ now means that $\boldsymbol{p}^{*}$ lies in the interior of $\mathcal{S}_{n-1}$. Thus we have the following elementary corollary to Theorem 1.

Corollary $\mathbf{1}$ If $W^{-1} \mathbf{1}>\mathbf{0}$ the minimum value of $w(\boldsymbol{p})$ for $\boldsymbol{p} \in \mathcal{S}_{n-1}$ occurs at the point $\boldsymbol{p}^{*}=$ $W^{-1} \mathbf{1} /\left(\mathbf{1}^{T} W^{-1} \mathbf{1}\right)$ in the interior of $\mathcal{S}_{n-1}$.

Remark 1 If the condition $W^{-1} \mathbf{1}>\mathbf{0}$ is not satisfied then the minimum value of $w(\boldsymbol{p})$ will occur on the boundary of $\mathcal{S}_{n-1}$. Let $\boldsymbol{k}=\{k(j)\}_{j=1}^{m}$ be an index set for some $m \in \mathbb{N}$ with $1 \leq m<n$ and $1 \leq k(1)<\cdots<k(m) \leq n$. Let $\mathcal{S}_{\boldsymbol{k}}=\left\{\boldsymbol{p} \mid \boldsymbol{p} \geq \mathbf{0}\right.$ with $\boldsymbol{p}^{T} \mathbf{1}=1$ and $p_{i}=0$ if $i \neq$ $k(j)$ for all $j=1, \ldots, m\}$ denote the corresponding face of the simplex $\mathcal{S}_{n-1}$. Note that $\mathcal{S}_{\boldsymbol{k}} \cong \mathcal{S}_{m-1}$. Define $\boldsymbol{p}_{\boldsymbol{k}}=\left[p_{k(j)}\right] \in \mathcal{S}_{m-1}, W_{\boldsymbol{k}}=\left[w_{k(i), k(j)}\right] \in \mathbb{R}^{m \times m}, \boldsymbol{w}_{\boldsymbol{k}}\left(\boldsymbol{p}_{\boldsymbol{k}}\right)=W_{\boldsymbol{k}} \boldsymbol{p}_{\boldsymbol{k}} \in \mathbb{R}^{m}$ and $w_{\boldsymbol{k}}\left(\boldsymbol{p}_{\boldsymbol{k}}\right)=$ $\boldsymbol{p}_{\boldsymbol{k}}{ }^{T} \boldsymbol{w}_{\boldsymbol{k}}\left(\boldsymbol{p}_{\boldsymbol{k}}\right)=\boldsymbol{p}_{\boldsymbol{k}}{ }^{T} W_{\boldsymbol{k}} \boldsymbol{p}_{\boldsymbol{k}} \in \mathbb{R}$. Now evolution of the population on $\mathcal{S}_{\boldsymbol{k}}$ can be described by the reduced replicator equation

$$
\boldsymbol{p}_{\boldsymbol{k}}(t+1)=\boldsymbol{p}_{\boldsymbol{k}}(t) \circ \boldsymbol{w}_{\boldsymbol{k}}(t) / w_{\boldsymbol{k}}(t)
$$


for all $t \in \mathbb{N}$ where we have written $\boldsymbol{w}_{\boldsymbol{k}}(t)=\boldsymbol{w}_{\boldsymbol{k}}\left[\boldsymbol{p}_{\boldsymbol{k}}(t)\right]$ and $w_{\boldsymbol{k}}(t)=w_{\boldsymbol{k}}\left[\boldsymbol{p}_{\boldsymbol{k}}(t)\right]$ for convenience. If $W_{\boldsymbol{k}}^{-1} \mathbf{1}>\mathbf{0}$ there is a local minimum for the fitness $w_{\boldsymbol{k}}(\boldsymbol{p})$ on the face $\mathcal{S}_{\boldsymbol{k}}$ at the point $\boldsymbol{p}_{\boldsymbol{k}}^{*}=$ $W_{\boldsymbol{k}}^{-1} \mathbf{1} /\left(\mathbf{1}^{T} W_{\boldsymbol{k}}^{-1} \mathbf{1}\right) \in \operatorname{int}\left(\mathcal{S}_{\boldsymbol{k}}\right)$. If $m>1$ then any such fixed points are unstable fixed points. The only possible stable fixed points in $\mathcal{S}_{n-1}$ are the vertices defined by $\boldsymbol{p}=\boldsymbol{e}_{j}$ for each $j=1, \ldots, n$. Every vertex of $\mathcal{S}_{n-1}$ is a fixed point but some vertices may not be stable. The vertex $\boldsymbol{e}_{j}$ is a stable fixed point if and only if the genotype $\mathcal{G}_{j}$ is conditionally dominant.

Remark 2 The condition that $W$ is positive definite is not necessary for Theorem 1. Define $V(t)=$ $\left[v_{i j}(t)\right] \in \mathbb{R}^{n \times n}$ by the formula $v_{i j}(t)=\sqrt{p_{i}}(t) w_{i j} \sqrt{p_{j}}(t)$ for $i, j=1, \ldots, n$, where $\boldsymbol{p}(t) \in \mathcal{S}_{n-1}$ for all $t \in \mathbb{N}$. It follows that $V(t)$ is symmetric with $v_{i j}(t) \geq 0$ for each $i, j=1, \ldots, n$. By using the symmetry of $W$ we have

$$
\begin{aligned}
& (\boldsymbol{p}(t) \circ \boldsymbol{w}(t))^{T} W(\boldsymbol{p}(t) \circ \boldsymbol{w}(t))=\sum_{i, j=1}^{n} p_{i}(t) w_{i}(t) \cdot w_{i j} \cdot p_{j}(t) w_{j}(t) \\
& \quad=\sum_{i, j=1}^{n} p_{i}(t) \cdot \sum_{k=1}^{n} p_{k}(t) w_{k i} \cdot w_{i j} \cdot \sum_{\ell=1}^{n} w_{j \ell} p_{\ell}(t) \cdot p_{j}(t) \\
& \quad=\sum_{k, \ell=1}^{n} \sqrt{p_{k}}(t) \cdot\left[\sum_{i, j=1}^{n} v_{k i}(t) \cdot v_{i j}(t) \cdot v_{j \ell}(t)\right] \cdot \sqrt{p_{\ell}}(t)=\sqrt{\boldsymbol{p}}(t)^{T} V(t)^{3} \sqrt{\boldsymbol{p}}(t) .
\end{aligned}
$$

From this identity and some elementary algebra it can be seen that

$$
w(t+1)-w(t)=\left[\sqrt{\boldsymbol{p}}(t)^{T} V(t)^{3} \sqrt{\boldsymbol{p}}(t)-\left(\sqrt{\boldsymbol{p}}(t)^{T} V(t) \sqrt{\boldsymbol{p}}(t)\right)^{3}\right] /\left(\sqrt{\boldsymbol{p}}(t)^{T} V(t) \sqrt{\boldsymbol{p}}(t)\right)^{2}
$$

for all $t \in \mathbb{N}$. We wish to show that $w(t+1)-w(t) \geq 0$. Let $V=\left[v_{i j}\right] \in \mathbb{R}^{n \times n}$ with $v_{i j}=v_{j i} \geq 0$ for all $i, j=1, \ldots, n$ and let $\boldsymbol{x}=\sqrt{\boldsymbol{p}}$ where $\boldsymbol{p} \in \mathcal{S}_{n-1}$. Therefore $\boldsymbol{x} \in \mathscr{S}_{n-1}^{+}=\left\{\boldsymbol{x} \in \mathbb{R}^{n} \mid \boldsymbol{x} \geq\right.$ $\mathbf{0}$ and $\|\boldsymbol{x}\|=1\}$. The set $\mathscr{S}_{n-1}^{+}$is a closed manifold in the orthant of the unit sphere $\mathscr{S}_{n-1} \subseteq \mathbb{R}^{n}$ defined by $\left\{\boldsymbol{e}_{i}\right\}_{i=1}^{n}$. Let

$$
\varphi(\boldsymbol{x})=\boldsymbol{x}^{T} V^{m} \boldsymbol{x}-\left(\boldsymbol{x}^{T} V \boldsymbol{x}\right)^{m}
$$

for some $m \in \mathbb{N}+1$ and every $\boldsymbol{x} \in \mathscr{S}_{n-1}^{+}$. Choose $P=\left[\boldsymbol{p}_{1}, \ldots, \boldsymbol{p}_{n}\right]$ so that $P^{T} V P=\Lambda=$ $\left[\lambda_{1} \boldsymbol{e}_{1}, \ldots, \lambda_{n} \boldsymbol{e}_{n}\right]$. Define $\boldsymbol{y}=P^{T} \boldsymbol{x} \in P^{T} \mathscr{S}_{n-1}^{+} \subseteq \mathbb{R}^{n}$. The set $P^{T} \mathscr{S}_{n-1}^{+}$is a closed manifold in the orthant of $\mathscr{S}_{n-1}$ defined by $\left\{\boldsymbol{f}_{i}\right\}_{i=1}^{n}=\left\{P^{T} \boldsymbol{e}_{i}\right\}_{i=1}^{n}$. Let

$$
\psi(\boldsymbol{y})=\boldsymbol{y}^{T} \Lambda^{m} \boldsymbol{y}-\left(\boldsymbol{y}^{T} \Lambda \boldsymbol{y}\right)^{m}=\sum_{j=1}^{n} \lambda_{j}^{m} y_{j}{ }^{2}-\left(\sum_{j=1}^{n} \lambda_{j} y_{j}^{2}\right)^{m}
$$

for $\boldsymbol{y} \in P^{T} \mathscr{S}_{n-1}^{+}$. We have $\psi(\boldsymbol{y})=\psi\left(P^{T} \boldsymbol{x}\right)=\varphi(\boldsymbol{x})$ for $\boldsymbol{x} \in \mathscr{S}_{n-1}^{+}$. Let $\boldsymbol{z}=\boldsymbol{y} \circ \boldsymbol{y}=Q(\boldsymbol{y})$ for $\boldsymbol{y} \in P^{T} \mathscr{S}_{n-1}^{+} \Leftrightarrow \boldsymbol{z} \in \mathscr{Q}=Q\left(P^{T} \mathscr{S}_{n-1}^{+}\right) \subseteq \mathcal{T}_{n-1}=\{\boldsymbol{z} \mid\langle\mathbf{1}, \boldsymbol{z}\rangle=1$ and $\boldsymbol{z} \geq \mathbf{0}\}$. Let

$$
\theta(\boldsymbol{z})=\sqrt{\boldsymbol{z}}^{T} \Lambda^{m} \sqrt{\boldsymbol{z}}-\left(\sqrt{\boldsymbol{z}}^{T} \Lambda \sqrt{\boldsymbol{z}}\right)^{m}=\sum_{j=1}^{n} \lambda_{j}^{m} z_{j}-\left(\sum_{j=1}^{n} \lambda_{j} z_{j}\right)^{m}
$$

for $\boldsymbol{z} \in \mathcal{T}_{n-1}$. Thus $\theta(\boldsymbol{z})=\varphi(\boldsymbol{x})$ for $\boldsymbol{z}=Q\left(P^{T} \boldsymbol{x}\right)$ and $\boldsymbol{x} \in \mathscr{S}_{n-1}^{+}$. Note that $\sum_{j=1}^{n} \lambda_{j} z_{j}=\boldsymbol{x}^{T} V \boldsymbol{x} \geq 0$ for $\boldsymbol{z} \in \mathscr{Q} \Leftrightarrow \boldsymbol{x} \in \mathscr{S}_{n-1}^{+}$. Differentiation now gives

$$
\partial^{2} \theta /\left[\partial z_{i} \partial z_{j}\right]=(-1) m(m-1)\left(\sum_{j=1}^{n} \lambda_{j} z_{j}\right)^{m-2} \lambda_{i} \lambda_{j}
$$

and the Hessian matrix is $H=(-1) m(m-1)\left(\sum_{j=1}^{n} \lambda_{j} z_{j}\right)^{m-2} \boldsymbol{\lambda} \boldsymbol{\lambda}^{T}$ where $\boldsymbol{\lambda}=\left[\lambda_{j}\right] \in \mathbb{R}^{n}$. Since $\left(\sum_{j=1}^{n} \lambda_{j} z_{j}\right)^{m-2} \geq 0$ it follows that $\boldsymbol{z}^{T} H \boldsymbol{z} \geq 0$ and so $\theta(\boldsymbol{z})$ is concave on $\mathscr{Q}$. For each $k \neq \ell$ there 
is an image $Z_{k \ell} \in \mathscr{Q}$ of a boundary segment for $P^{T} \mathscr{S}_{n-1}^{+} \subseteq \mathscr{S}_{n-1}$ with $\boldsymbol{z}=\left(\boldsymbol{f}_{k} \circ \boldsymbol{f}_{k}\right) \cos ^{2} \alpha+2\left(\boldsymbol{f}_{k} \circ\right.$ $\left.\boldsymbol{f}_{\ell}\right) \cos \alpha \sin \alpha+\left(\boldsymbol{f}_{\ell} \circ \boldsymbol{f}_{\ell}\right) \sin ^{2} \alpha$ where $\theta(\boldsymbol{z})$ is given by

$$
\theta_{k \ell}(\sigma)=\lambda_{k}{ }^{m} \sigma+\lambda_{\ell}{ }^{m}(1-\sigma)-\left[\lambda_{k} \sigma+\lambda_{\ell}(1-\sigma)\right]^{m}
$$

and $\sigma=\cos ^{2} \alpha \in[0,1] \Leftrightarrow \alpha \in[0, \pi / 2]$. Since $\theta_{k \ell}(\sigma)$ is concave for $\sigma \in[0,1]$ with $\theta_{k \ell}(0)=\varphi\left(\boldsymbol{e}_{k}\right) \geq 0$ and $\theta_{k \ell}(1)=\varphi\left(\boldsymbol{e}_{\ell}\right) \geq 0$ we have $\theta_{k \ell}(\sigma) \geq 0$ for all $\sigma \in[0,1] \Leftrightarrow \boldsymbol{z} \in Z_{k \ell}$. Due to potential folding caused by the mapping $Q$ at the boundary of $\mathcal{T}_{n-1}$, we must also show that $\theta(\boldsymbol{z}) \geq 0$ on certain boundary segments of $\mathcal{T}_{n-1}$ to complete a geometric proof that $\theta(\boldsymbol{z}) \geq 0$ for all $\boldsymbol{z} \in \mathscr{Q}$. This is a non-trivial task. Nevertheless the above arguments give an intuitive idea of what is required. See the original article [16] for a complete proof.

Example 1 Let

$$
W=\left[\begin{array}{ccc}
3 & 2 & 4 \\
2 & 5 & 4 \\
4 & 4 & 7
\end{array}\right] \in \mathbb{R}^{3 \times 3}
$$

According to MATLAB the symmetric matrix $W$ is positive definite with eigenvalues given approximately by $\boldsymbol{\lambda} \approx(0.4589,2.3174,12.2237)$. The inverse matrix is

$$
W^{-1}=\left[\begin{array}{rrr}
19 / 13 & 2 / 13 & -12 / 13 \\
2 / 13 & 5 / 13 & -4 / 13 \\
-12 / 13 & -4 / 14 & 11 / 13
\end{array}\right]
$$

and so

$$
W^{-1} \mathbf{1}=\left[\begin{array}{r}
9 / 13 \\
3 / 13 \\
-5 / 13
\end{array}\right] \nsupseteq \mathbf{0} .
$$

Hence there is no minimum point for the population fitness in $\operatorname{int}\left(\mathcal{S}_{2}\right)$. However we note that

$$
\begin{gathered}
W_{\{1,2\}}=\left[\begin{array}{ll}
3 & 2 \\
2 & 5
\end{array}\right] \in \mathbb{R}^{2 \times 2} \Rightarrow W_{\{1,2\}}^{-1}=\left[\begin{array}{rr}
5 / 11 & -2 / 11 \\
-2 / 11 & 3 / 11
\end{array}\right], \\
W_{\{1,3\}}=\left[\begin{array}{ll}
3 & 4 \\
4 & 7
\end{array}\right] \in \mathbb{R}^{2 \times 2} \Rightarrow W_{\{1,3\}}^{-1}=\left[\begin{array}{rr}
7 / 5 & -4 / 5 \\
-4 / 5 & 3 / 5
\end{array}\right]
\end{gathered}
$$

and

$$
W_{\{2,3\}}=\left[\begin{array}{ll}
5 & 4 \\
4 & 7
\end{array}\right] \in \mathbb{R}^{2 \times 2} \Rightarrow W_{\{2,3\}}^{-1}=\left[\begin{array}{rr}
7 / 19 & -4 / 19 \\
-4 / 19 & 5 / 19
\end{array}\right] \text {. }
$$

Therefore

$$
W_{\{1,2\}}^{-1} \mathbf{1}=\left[\begin{array}{l}
3 / 11 \\
1 / 11
\end{array}\right]>\mathbf{0} \quad \text { and } \quad W_{\{2,3\}}^{-1} \mathbf{1}=\left[\begin{array}{c}
3 / 19 \\
1 / 19
\end{array}\right]>\mathbf{0} .
$$

Hence there are minimum points for the population fitness on $\mathcal{S}_{\{1,2\}}$ at

$$
\boldsymbol{p}_{\{1,2\}}^{*}=W_{\{1,2\}}^{-1} \mathbf{1} /\left(\mathbf{1}^{T} W_{\{1,2\}}^{-1} \mathbf{1}\right)=\left[\begin{array}{c}
3 / 4 \\
1 / 4 \\
0
\end{array}\right] \in \operatorname{int}\left(\mathcal{S}_{\{1,2\}}\right) \cong \mathcal{S}_{1}
$$


and on $\mathcal{S}_{\{2,3\}}$ at

$$
\boldsymbol{p}_{\{2,3\}}^{*}=W_{\{2,3\}}^{-1} \mathbf{1} /\left(\mathbf{1}^{T} W_{\{2,3\}}^{-1} \mathbf{1}\right)=\left[\begin{array}{c}
0 \\
3 / 4 \\
1 / 4
\end{array}\right] \in \operatorname{int}\left(\mathcal{S}_{\{2,3\}}\right) \cong \mathcal{S}_{1}
$$

There is no minimum point on $\mathcal{S}_{\{1,3\}}$. The level curves for the fitness are shown in Figure 1 . The global minimum fitness is at $\boldsymbol{p}_{\{1,2\}}^{*} \in \operatorname{int}\left(\mathcal{S}_{\{1,2\}}\right)$.

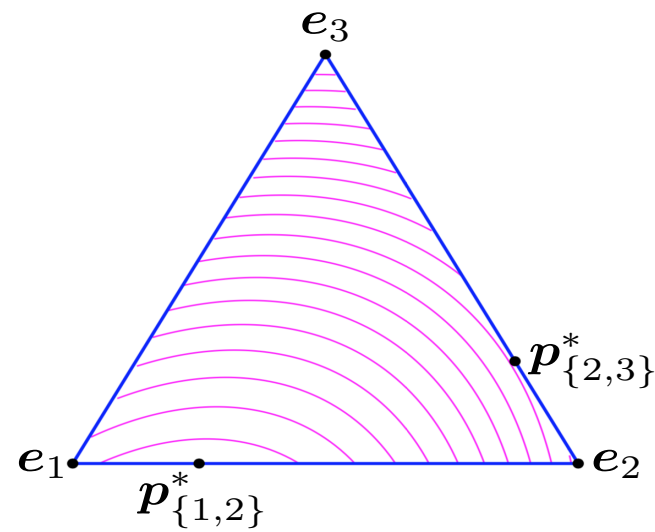

Figure 1: Level curves for the population fitness $w(\boldsymbol{p})$ in Example 1 showing the unstable fixed points $\boldsymbol{p}_{\{1,2\}}^{*} \in \operatorname{int}\left(\mathcal{S}_{\{1,2\}}\right)$ and $\boldsymbol{p}_{\{2,3\}}^{*} \in \operatorname{int}\left(\mathcal{S}_{\{2,3\}}\right)$. The vertex $\boldsymbol{e}_{1}$ is an unstable (quasi-stable) fixed point and the vertices $\boldsymbol{e}_{2}$ and $\boldsymbol{e}_{3}$ are stable fixed points.

Example 2 Let

$$
W=\left[\begin{array}{lll}
19 & 12 & 10 \\
12 & 17 & 10 \\
10 & 10 & 18
\end{array}\right]
$$

The unstable fixed points are

$$
\begin{gathered}
\boldsymbol{p}_{\{1,2,3\}}^{*}=W^{-1} \mathbf{1} /\left(\mathbf{1}^{T} W^{-1} \mathbf{1}\right) \approx\left[\begin{array}{l}
0.2581 \\
0.3613 \\
0.3806
\end{array}\right] \in \operatorname{int}\left(\mathcal{S}_{2}\right), \quad \boldsymbol{p}_{\{1,2\}}^{*}=\left[\begin{array}{c}
5 / 12 \\
7 / 12 \\
0
\end{array}\right] \in \operatorname{int}\left(\mathcal{S}_{\{1,2\}}\right), \\
\boldsymbol{p}_{\{1,3\}}^{*}=\left[\begin{array}{c}
8 / 17 \\
0 \\
9 / 17
\end{array}\right] \in \operatorname{int}\left(\mathcal{S}_{\{1,3\}}\right) \quad \text { and } \boldsymbol{p}_{\{2,3\}}^{*}=\left[\begin{array}{c}
0 \\
8 / 15 \\
7 / 15
\end{array}\right] \in \operatorname{int}\left(\mathcal{S}_{\{2,3\}}\right) .
\end{gathered}
$$

The stable fixed points are the vertices $\boldsymbol{e}_{1}, \boldsymbol{e}_{2}$ and $\boldsymbol{e}_{3}$. Figure 2 shows a selection of evolutionary paths starting near the minimum fitness point $\boldsymbol{p}_{\{1,2,3\}}^{*} \in \operatorname{int}\left(\mathcal{S}_{2}\right)$. 


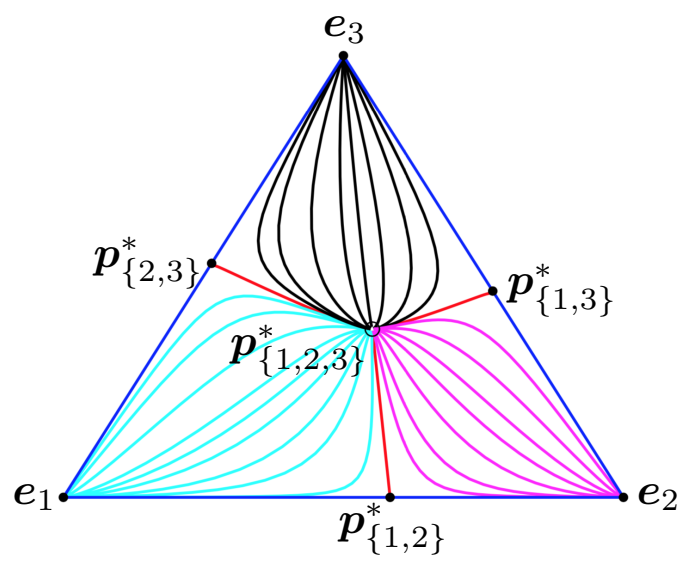

Figure 2: Evolutionary paths for Example 2 starting near the unstable fixed point $\boldsymbol{p}_{\{1,2,3\}}^{*} \in \operatorname{int}\left(\mathcal{S}_{2}\right)$ showing unstable fixed points $\boldsymbol{p}_{\{1,2\}}^{*} \in \operatorname{int}\left(\mathcal{S}_{\{1,2\}}\right), \boldsymbol{p}_{\{1,3\}}^{*} \in \operatorname{int}\left(\mathcal{S}_{\{1,3\}}\right)$ and $\boldsymbol{p}_{\{2,3\}}^{*} \in \operatorname{int}\left(\mathcal{S}_{\{2,3\}}\right)$. The vertices $\boldsymbol{e}_{1}, \boldsymbol{e}_{2}$ and $\boldsymbol{e}_{3}$ are all stable fixed points.

\section{A special case of population evolution in discrete time}

To introduce the key ideas underlying discrete-time evolution with random perturbations we begin by discussing a special case that serves as an elementary prototype. We consider a population evolution of two genotypes defined by the replicator equation (2) in the special case where

$$
W=\left[\begin{array}{cc}
\alpha^{2} & 0 \\
0 & 1
\end{array}\right] \in \mathbb{R}^{2 \times 2} \quad \text { and } \quad \boldsymbol{p}(t)=\left[\begin{array}{c}
p(t) \\
1-p(t)
\end{array}\right] \in \mathcal{S}_{1}
$$

for all $t \in \mathbb{N}$ where $\alpha \in \mathbb{R}$ with $\alpha>0$. We need only consider the first component of $\boldsymbol{p}$. We have

$$
p(t+1)=\alpha^{2} p(t)^{2} /\left[\alpha^{2} p(t)^{2}+(1-p(t))^{2}\right]
$$

with $0 \leq p(1) \leq 1$. Suppose the population at time $t$ is disrupted by random changes to the relative genotype frequencies. Thus we assume that the population at time $t$ is perturbed by the addition of a small multiple of a randomly generated population of the same two genotypes. Specifically we suppose that (5) is replaced by

$$
p(t+1)=\frac{\alpha^{2}[(1-\epsilon) p(t)+\epsilon r(t)]^{2}}{\alpha^{2}[(1-\epsilon) p(t)+\epsilon r(t)]^{2}+(1-[(1-\epsilon) p(t)+\epsilon r(t)])^{2}}
$$

for all $t \in \mathbb{N}$ where $\boldsymbol{r}=\{r(t)\}_{t \in \mathbb{N}} \in[0,1]^{\infty}$ is an outcome generated by a sequence of independent identically distributed random variables defined by a cumulative distribution function $\mathcal{F}: \mathbb{R} \rightarrow$ $[0,1]$. For each finite collection of distinct times $\left\{t_{k}\right\}_{k=1}^{s} \subseteq \mathbb{N}$ we will suppose that the cumulative distribution function $\mathcal{F}_{t_{1}, \ldots, t_{s}}: \mathbb{R}^{s} \rightarrow[0,1]^{s}$ takes the form $\mathcal{F}_{t_{1}, t_{2}, \ldots, t_{s}}\left(x_{1}, x_{2}, \ldots, x_{s}\right)=\prod_{k=1}^{s} \mathcal{F}\left(x_{k}\right)$ 
where $\mathcal{F}: \mathbb{R} \rightarrow[0,1]$ is a right-continuous strictly increasing function with $\mathcal{F}(x)=0$ for $x<0$, $\mathcal{F}(x) \in(0,1)$ for $x \in(0,1)$ and $\mathcal{F}(x)=1$ for $x \geq 1$. The right continuity means that $\mathcal{F}(0)=$ $\lim _{x \downarrow 0} \mathcal{F}(x)$. The function $\mathcal{F}$ may have a jump discontinuity on the left at any point $x \in[0,1]$. We could consider an apparently simpler evolution defined by

$$
p(t+1)=(1-\epsilon) \alpha^{2} p(t)^{2} /\left[\alpha^{2} p(t)^{2}+(1-p(t))^{2}\right]+\epsilon r(t+1)
$$

for all $t \in \mathbb{N}$. However the simplification is largely illusory. At each stage the first form (6) adds noise and then evolves deterministically whereas the second form (7) evolves deterministically and then adds noise. The two forms are essentially equivalent. In the first case we perturb the current population and then evolve deterministically to the new population. In the second case we evolve deterministically from the current population to the new population and then perturb the new population. In each case the two processes alternate: perturb and evolve, or evolve and perturb, repeated ad infinitum. In order to study the distribution of the unperturbed sequence $\{p(t)\}_{t \in \mathbb{N}}$ with $\epsilon=0$ it is useful to consider the equation

$$
\alpha^{2} y^{2} /\left[\alpha^{2} y^{2}+(1-y)^{2}\right]=x .
$$

We will show that for each $x \in[0,1]$ this equation has a unique solution $y \in[0,1]$. To begin consider the function $F(y)=F_{\alpha}(y)$ defined by

$$
F_{\alpha}(y)=\alpha^{2} y^{2} /\left[\alpha^{2} y^{2}+(1-y)^{2}\right]
$$

for $y \in[0,1]$. We use elementary calculus to show $F(y)$ is a cumulative distribution function with corresponding density $f(y)=F^{\prime}(y)$. The maximum density occurs when $f^{\prime}(y)=F^{\prime \prime}(y)=0$ which gives

$$
2\left(1+\alpha^{2}\right) y^{3}-3\left(1+\alpha^{2}\right) y^{2}+1=0 \Longleftrightarrow z^{3}-3\left(1+\alpha^{2}\right) z+2\left(1+\alpha^{2}\right)=0
$$

where we have defined $z=1 / y$. This is a cubic equation with no quadratic term. We apply a standard solution procedure. The substitution $z=r \sin \theta$ and the application of a standard trigonometric triple-angle formula gives

$$
\left(r^{3} / 4\right) \sin 3 \theta+\left[3 r\left(1+\alpha^{2}\right)-3 r^{3} / 4\right] \sin \theta-2\left(1+\alpha^{2}\right)=0 .
$$

We can eliminate the term in $\sin \theta$ by choosing $r=2 \sqrt{1+\alpha^{2}}$ to give

$$
\sin 3 \theta=\frac{1}{\sqrt{1+\alpha^{2}}} \Longleftrightarrow \theta=\frac{1}{3} \arcsin \left(\frac{1}{\sqrt{1+\alpha^{2}}}\right)+\frac{2 \pi k}{3}
$$

for $k=0,1,2$. Since $y=1 /(r \sin \theta)$ the maximum turning point occurs when

$$
y=1 /\left(2 \sqrt{1+\alpha^{2}} \sin \left[(1 / 3) \arcsin \left(1 / \sqrt{1+\alpha^{2}}\right)+2 \pi / 3\right]\right) .
$$

The cumulative distribution function $F(y)$ and associated probability density function $f(y)=F^{\prime}(y)$ are plotted in Figure 3 for $\alpha^{2}=0.25,1,4$. 

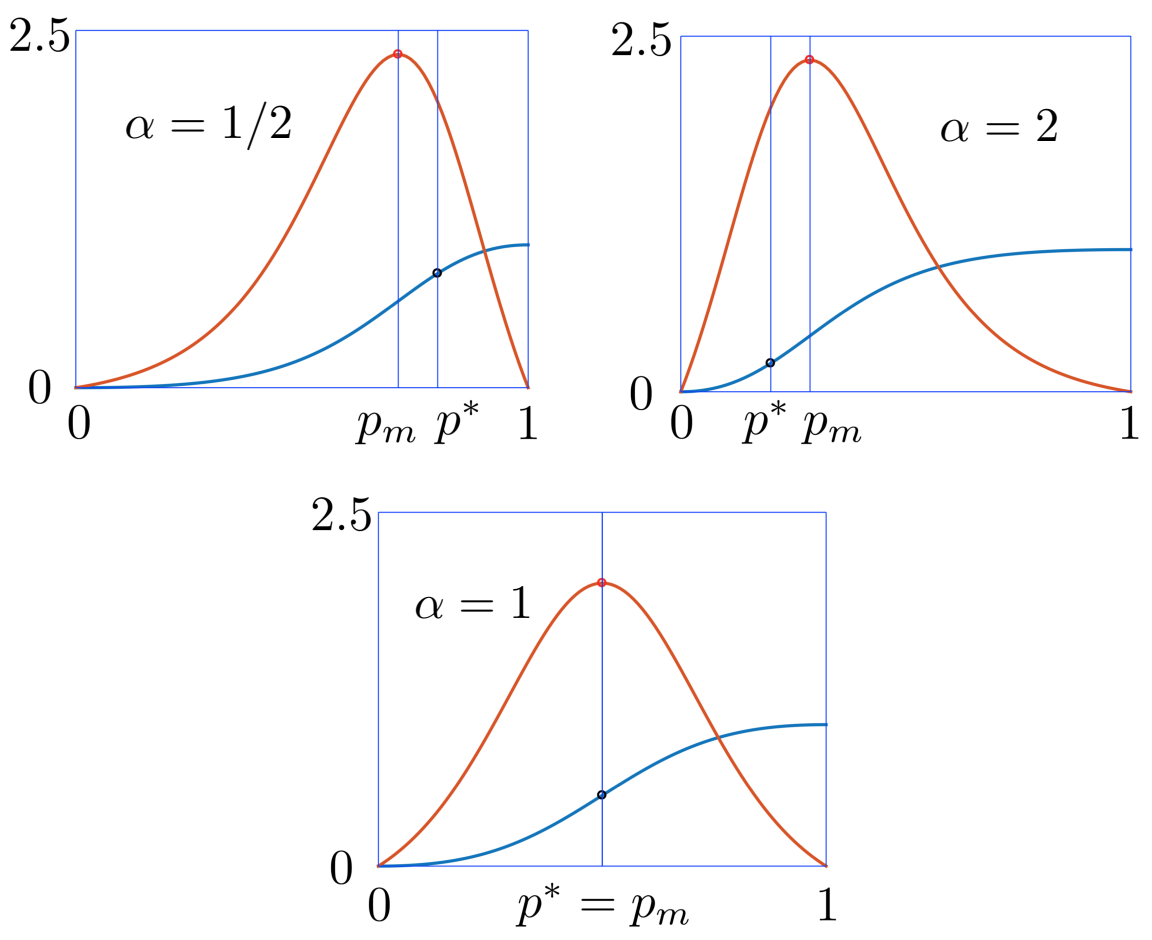

Figure 3: The cumulative distribution function $F(y)$ (blue curve) and associated probability density function $f(y)=F^{\prime}(y)$ (red curve) for the one-dimensional evolution in Section 5 with $\alpha=1 / 2$ (top left), $\alpha=2$ (top right) and $\alpha=1$ (bottom) showing the unstable interior fixed point $p^{*}=1 /\left(1+\alpha^{2}\right)$ and the maximum point $p_{m}=1 /\left[2 \sqrt{1+\alpha^{2}} \sin \left(\arcsin \left(1 / \sqrt{1+\alpha^{2}}\right) / 3+2 \pi / 3\right)\right]$ for the density function.

The strictly monotone nature of $F(y)$ means that (8) has a unique solution $y=F^{-1}(x) \in[0,1]$ for each $x \in[0,1]$. If we define $x=\sin ^{2} \psi$ where $\psi \in[0, \pi / 2]$ then some elementary algebra shows that $x=F(x) \Leftrightarrow \sin ^{2} \psi=\sin \psi /(\sin \psi+\alpha \cos \psi) \Leftrightarrow \tan \psi=1 / \alpha \Leftrightarrow \sin \psi=1 / \sqrt{1+\alpha^{2}} \Leftrightarrow x=1 /\left(1+\alpha^{2}\right)$. Thus $p^{*}=1 /\left(1+\alpha^{2}\right)$ is also a fixed point. We wish to find out what happens when we iterate the map. For the unperturbed map there are two stable regions. We have $F(y)<y$ when $0<y<p^{*}$ and $F(y)>y$ when $p^{*}<y<1$. Thus, if we define the intervals $I=\left[0, p^{*}\right)$ and $J=\left(p^{*}, 1\right]$, then we can see that $F(I) \subset I$ and $F(J) \subset J$.

\subsection{The iterated maps}

Let $\epsilon \in[0,1]$ be the perturbation parameter and let $\boldsymbol{r}=\{r(t)\}_{t \in \mathbb{N}} \in[0,1]^{\infty}$ be an outcome generated by a sequence of independent identically distributed random variables. Define $F_{\alpha, \epsilon, \boldsymbol{r}}^{(t)}:[0,1] \rightarrow[0,1]$ by setting

$$
F_{\alpha, \epsilon, r}^{(1)}(x)=F_{\alpha}[(1-\epsilon) x+\epsilon r(1)]
$$


and for each $t \in \mathbb{N}$ define $F_{\alpha, \epsilon, \boldsymbol{r}}^{(t+1)}:[0,1] \rightarrow[0,1]$ by the recursive formula

$$
F_{\alpha, \epsilon, \boldsymbol{r}}^{(t+1)}(x)=F_{\alpha}\left[(1-\epsilon) F_{\alpha, \epsilon, \boldsymbol{r}}^{(t)}(x)+\epsilon r(t+1)\right]
$$

where $F_{\alpha}:[0,1] \rightarrow[0,1]$ is defined in (9). When the context is clear we may suppress the dependence on one or more of the parameters $\alpha, \epsilon$ and $\boldsymbol{r}$.

\subsubsection{The iterated unperturbed map.}

Consider the iterated unperturbed map with $\epsilon=0$. The fixed point $p^{*}=1 /\left(1+\alpha^{2}\right)$ is an unstable fixed point while the other two fixed points 0 and 1 are stable fixed points. It follows that $F^{(t)}(x) \downarrow 0$ if $x \in\left(0, p^{*}\right)$ and $F^{(t)}(x) \uparrow 1$ if $x \in\left(p^{*}, 1\right)$ as $t \uparrow \infty$. Therefore we have a pointwise limit function

$$
F^{(\infty)}(x)= \begin{cases}0 & \text { for } 0 \leq x<p^{*} \\ p^{*} & \text { for } x=p^{*} \\ 1 & \text { for } p^{*}<x \leq 1\end{cases}
$$

For each particular point $x(1) \in[0,1]$ the iterated unperturbed map defines an evolution $\{x(t)\}_{t \in \mathbb{N}}$ starting from $x(1)$ by the formula $x(t+1)=F^{(t)}[x(1)]$ for each $t \in \mathbb{N}$. The iterated unperturbed maps are shown in Figure 4.

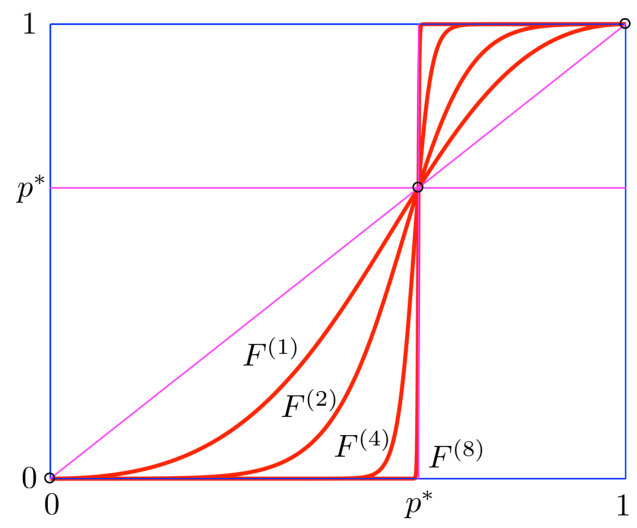

Figure 4: The iterated unperturbed maps $y=F^{(t)}(x)$ for $t=1,2,4,8$ when $\alpha=0.75$.

\subsubsection{The iterated perturbed map.}

For the perturbed map with $\epsilon \in(0,1]$ starting from $x(1)$ we can think of the evolution as a two-stage process with

$$
y(t)=(1-\epsilon) x(t)+\epsilon r(t)
$$


where $\boldsymbol{r}=\{r(t)\}_{t \in \mathbb{N}} \in[0,1]^{\infty}$ is an outcome generated by a sequence of independent identically distributed random variables and $x(t+1)=F[y(t)]$ for each $t \in \mathbb{N}$. We know that $|y(t)-x(t)|=$ $\epsilon|r(t)-x(t)| \leq \epsilon$ and so $y(t)$ is uniformly close to $x(t)$. If $\epsilon>0$ is sufficiently small we might wonder if the iterated perturbed map converges approximately in some as yet unspecified way to a limiting perturbed map that is close to the limiting unperturbed map.

In Figure 5 we set $s=1500$ and $\epsilon=0.1$ and show the graphs of $y=F_{\alpha, \epsilon, r}^{(s)}(x)$ corresponding to 1000 different pseudo-random sequences $\boldsymbol{r}=\{r(t)\}_{t=1}^{s} \in[0,1]^{s}$ for $\alpha=0.75,1,1.4$ generated in MAтLAв by a sequence of independent uniformly distributed pseudo-random variables. For each $\boldsymbol{r}$ the graph $y=F_{\alpha, \epsilon, r}^{(s)}(x)$ is an approximate step function with a uniquely defined jump point $p_{\boldsymbol{r}}$ and with $\lim \sup _{x \downarrow 0} F_{\alpha, \epsilon, r}^{(s)}(x)=\xi_{1}$ and $\liminf _{x \uparrow 1} F_{\alpha, \epsilon, r}^{(s)}(x)=\eta_{1}$. For each value of $\alpha$ we have highlighted (in red) a typical graph $y=F_{\alpha, \epsilon, r^{*}}^{(s)}(x)$ for one particular value of $\boldsymbol{r}=\boldsymbol{r}^{*} \in[0,1]^{s}$ and have also graphed (in black) the average over all $\boldsymbol{r} \in[0,1]^{s}$ of the individual graphs. In the context of a recursive map both axes are essentially the same. The interval $\left[\xi_{0}, \eta_{0}\right]$ is shown on the horizontal $x$-axis, because the jump point $p_{\boldsymbol{r}}$ lies in this interval for all pseudo-random sequences $\boldsymbol{r}$. The fixed point $p^{*}$ for the unperturbed map also lies in this interval. The points $\xi_{1}$ and $\eta_{1}$ are shown on the vertical $y$-axis because for each $\boldsymbol{r}$ the values of the approximate step functions are eventually captured in the interval $\left[0, \xi_{1}\right]$ when $x<p_{\boldsymbol{r}}$ and in the interval $\left[\eta_{1}, 1\right]$ when $x>p_{\boldsymbol{r}}$. Although we have used a uniform distribution in this case, we note that similar results are obtained for many other distributions. We have the following key result.

Theorem 2 Let $\boldsymbol{r}=\{r(t)\}_{t \in \mathbb{N}} \in[0,1]^{\infty}$ be an arbitrary sequence either random or deterministic - and suppose that $x(1) \in[0,1]$ and that the perturbed mapping $\{x(t)\}_{t \in \mathbb{N}} \in[0,1]^{\infty}$ is defined by $y(t)=(1-\epsilon) x(t)+\epsilon r(t)$ and $x(t+1)=F_{\alpha}[y(t)]$ for all $t \in \mathbb{N}$ where $\alpha \in(0,1)$ is a constant and $\epsilon \in(0,1)$ is a perturbation parameter. If $p^{*}=1 /\left(1+\alpha^{2}\right)$ is the fixed point for the unperturbed mapping and if $\epsilon$ is sufficiently small to ensure that $2 \sqrt{\epsilon} /(1-\epsilon)<\alpha<(1-\epsilon) /(2 \sqrt{\epsilon})$, then there exist proliferation intervals $\left[\eta_{0}(\epsilon), \eta_{1}(\epsilon)\right] \subset\left(p^{*}, 1\right)$ such that $x(t+1) \geq x(t)$ if $x(t) \in\left[\eta_{0}(\epsilon), \eta_{1}(\epsilon)\right]$ and $\left[\xi_{1}(\epsilon), \xi_{0}(\epsilon)\right] \subset\left(0, p^{*}\right)$ such that $x(t+1) \leq x(t)$ if $x(t) \in\left[\xi_{1}(\epsilon), \xi_{0}(\epsilon)\right]$. There also exist associated capture intervals $\left[\eta_{0}(\epsilon), 1\right]$ and $\left[0, \xi_{0}(\epsilon)\right]$ such that if $x(t) \in\left[\eta_{0}(\epsilon), \eta_{1}(\epsilon)\right]$, then we must have $x(s) \in\left[\eta_{0}(\epsilon), 1\right]$ for all $s \geq t$, and if $x(t) \in\left[\xi_{1}(\epsilon), \xi_{0}(\epsilon)\right]$, then we must have $x(s) \in\left[0, \xi_{0}(\epsilon)\right]$ for all $s \geq t$.

Proof For the moment we write $F_{\alpha}(y)=\alpha^{2} y^{2} /\left[\alpha^{2} y^{2}+(1-y)^{2}\right]$ for each $y \in[0,1]$. Define $\beta=1 / \alpha$ and note that $1-F_{\alpha}(y)=F_{\beta}(1-y)$ for all $y \in[0,1]$. Therefore

$$
F_{\alpha}(y)+F_{\beta}(1-y)=1
$$

for all $y \in[0,1]$. Let $\boldsymbol{q}=\mathbf{1}-\boldsymbol{r} \Longleftrightarrow\{q(t)\}_{t \in \mathbb{N}}=\{1-r(t)\}_{t \in \mathbb{N}} \in[0,1]^{\infty}, \boldsymbol{u}=\mathbf{1}-\boldsymbol{x} \Longleftrightarrow\{u(t)\}_{t \in \mathbb{N}}=$ $\{1-x(t)\}_{t \in \mathbb{N}}$ and $\boldsymbol{v}=\mathbf{1}-\boldsymbol{y} \Longleftrightarrow\{v(t)\}_{t \in \mathbb{N}}=\{1-y(t)\}_{t \in \mathbb{N}}$. Now

$$
\begin{aligned}
\boldsymbol{y}=(1-\epsilon) \boldsymbol{x}+\epsilon \boldsymbol{r} & \Longleftrightarrow y(t)=(1-\epsilon) x(t)+\epsilon r(t) \\
& \Longleftrightarrow v(t)=(1-\epsilon) u(t)+\epsilon q(t) \Longleftrightarrow \boldsymbol{v}=(1-\epsilon) \boldsymbol{u}+\epsilon \boldsymbol{q}
\end{aligned}
$$



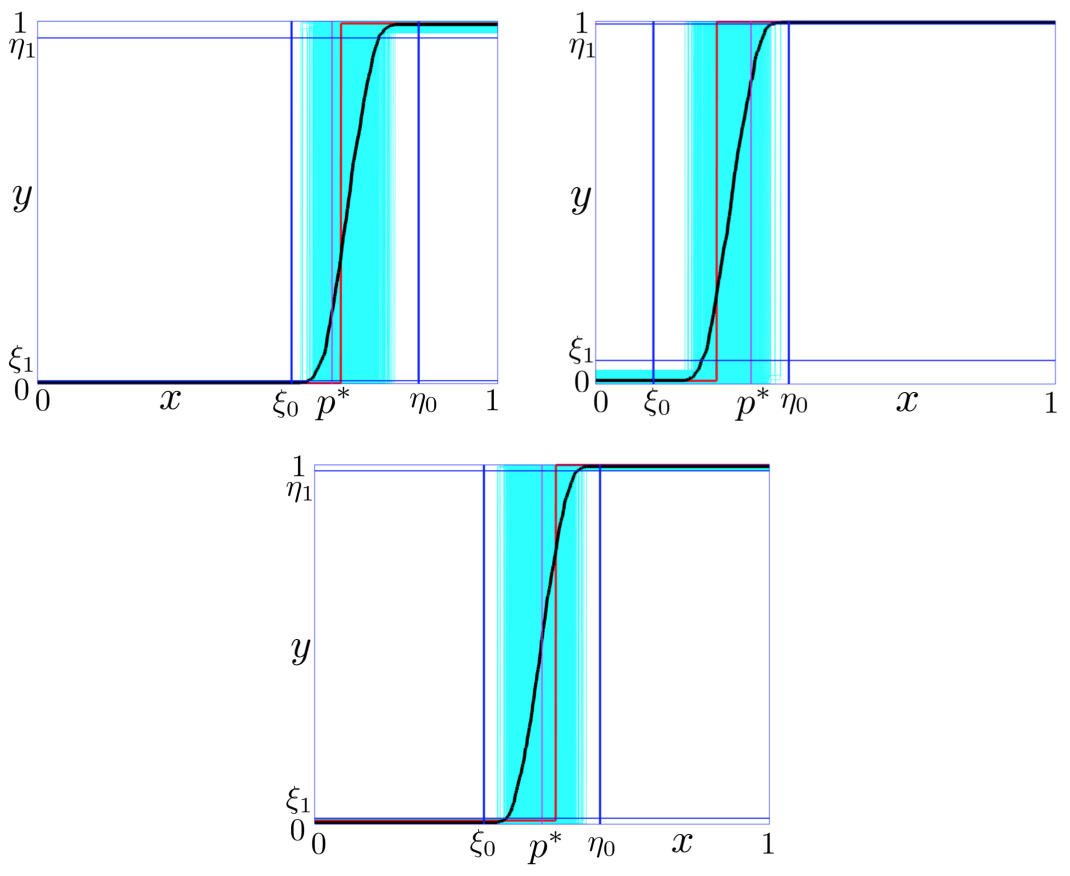

Figure 5: Each of the 1000 iterated perturbed maps (thin blue lines) defined by $y=F_{\alpha, \epsilon, r}^{s}(x)$ for $s=1500$ is an approximate step function, with $\lim \sup _{x \downarrow 0} F_{\alpha, \epsilon, \boldsymbol{r}}^{s}(x)=\xi_{1}$ and $\liminf \operatorname{in}_{x \uparrow 1} F_{\alpha, \epsilon, \boldsymbol{r}}^{s}(x)=\eta_{1}$, and with jump point $p_{\boldsymbol{r}} \in\left(\xi_{0}, \eta_{0}\right)$. A typical approximate step function for one particular outcome $\boldsymbol{r}=\boldsymbol{r}^{*} \in[0,1]^{s}$ (medium red line) is highlighted and the average limiting map over all outcomes $\boldsymbol{r} \in[0,1]^{s}$ (thick black line) is also highlighted for $\alpha=0.75$ on the top left, $\alpha=1.4$ on the top right, and $\alpha=1$ on the bottom, with $\epsilon=0.1$ in each case. The jump interval $\left[\xi_{0}, \eta_{0}\right]$ and the superior and inferior limits $\xi_{1}$ and $\eta_{1}$ are shown on the horizontal and vertical axes respectively. The fixed point $p^{*}$ for the unperturbed map lies in the interval $\left[\xi_{0}, \eta_{0}\right]$.

and hence

$$
x(t+1)=F_{\alpha}[y(t)] \Longleftrightarrow u(t+1)=F_{\beta}[v(t)] .
$$

We assume that $\epsilon \in(0,1)$ is fixed. It turns out that the key values $\xi_{j}=\xi_{j}(\epsilon)$ and $\eta_{j}=\eta_{j}(\epsilon)$ for each $j=0,1$ all depend on $\epsilon$. We will often omit this dependence in order to avoid excessively complex notation. The complementary relationship between $F_{\alpha}$ and $F_{\beta}$ can be used to show that the problem of finding $\left[\xi_{1}, \xi_{0}\right]$ is essentially the same as the problem of finding $\left[\eta_{0}, \eta_{1}\right]$. We wish to find $\left[\eta_{0}, \eta_{1}\right]$ such that $F_{\alpha}[(1-\epsilon) \eta+\epsilon r] \geq \eta$ for all $\eta \in\left[\eta_{0}, \eta_{1}\right]$ irrespective of $r \in[0,1]$. The worst case is when $r=0$. Thus we must consider the inequality $F_{\alpha}[(1-\epsilon) \eta] \geq \eta$. We also wish to find $\left[\xi_{1}, \xi_{0}\right]$ such that $F_{\alpha}[(1-\epsilon) \xi+\epsilon r] \leq \xi$ for all $\xi \in\left[\xi_{1}, \xi_{0}\right]$ irrespective of $r \in[0,1]$. This time the worst case is $r=1$ so we must consider the inequality $F_{\alpha}[(1-\epsilon) \xi+\epsilon] \leq \xi$.

Firstly consider the inequality $F_{\alpha}[(1-\epsilon) \eta] \geq \eta \Longleftrightarrow F_{\alpha}(\theta) \geq \theta /(1-\epsilon)$ where $\theta=(1-\epsilon) \eta>0$. 
Therefore

$$
\frac{\alpha^{2} \theta^{2}}{\alpha^{2} \theta^{2}+(1-\theta)^{2}} \geq \frac{\theta}{(1-\epsilon)} \Longleftrightarrow \theta^{2}-\frac{2+(1-\epsilon) \alpha^{2}}{1+\alpha^{2}} \theta+\frac{1}{1+\alpha^{2}} \leq 0
$$

Now

$$
\theta^{2}-\frac{2+(1-\epsilon) \alpha^{2}}{1+\alpha^{2}} \theta+\frac{1}{1+\alpha^{2}}=0
$$

if and only if

$$
\theta=\frac{1+(1-\epsilon) \alpha^{2} / 2 \pm \sqrt{\left[1+(1-\epsilon) \alpha^{2} / 2\right]^{2}-\left(1+\alpha^{2}\right)}}{1+\alpha^{2}}
$$

These solutions are real if and only if $\alpha \geq 2 \sqrt{\epsilon} /(1-\epsilon)$. This condition is certainly true if $\epsilon$ is sufficiently small in which case we denote the roots by

$$
\theta_{0}(\epsilon)=\frac{1+(1-\epsilon) \alpha^{2} / 2-\alpha \sqrt{(1-\epsilon)^{2} \alpha^{2} / 4-\epsilon}}{1+\alpha^{2}}
$$

and

$$
\theta_{1}(\epsilon)=\frac{1+(1-\epsilon) \alpha^{2} / 2+\alpha \sqrt{(1-\epsilon)^{2} \alpha^{2} / 4-\epsilon}}{1+\alpha^{2}} .
$$

Since $\theta_{0}(\epsilon) \theta_{1}(\epsilon)=1 /\left(1+\alpha^{2}\right)$ we have

$$
\theta_{0}(\epsilon)=\frac{1}{1+(1-\epsilon) \alpha^{2} / 2+\alpha \sqrt{(1-\epsilon)^{2} \alpha^{2} / 4-\epsilon}} .
$$

Now we define the corresponding solutions for $\eta=\theta /(1-\epsilon)$ by setting $\eta_{j}(\epsilon)=\theta_{j}(\epsilon) /(1-\epsilon)$ for each $j=0,1$. If $\epsilon$ is small we have

$$
\eta_{0}(\epsilon) \approx \eta_{0}(0)+\eta_{0}^{\prime}(0) \epsilon=1 /\left(1+\alpha^{2}\right)+2 \epsilon /\left(1+\alpha^{2}\right)
$$

and, since $\eta_{1}^{\prime}(0)=0$, we also have

$$
\eta_{1}(\epsilon) \approx \eta_{1}(0)+\eta_{1}^{\prime \prime}(0) \epsilon^{2} / 2=1-\epsilon^{2} / \alpha^{2} .
$$

If $x(s) \in\left(\eta_{0}, \eta_{1}\right)$ then $x(s+1)=F_{\alpha}[(1-\epsilon) x(s)+\epsilon r(s)] \geq F_{\alpha}[(1-\epsilon) x(s)] \geq x(s)$. Thus $x(s)$ is non-decreasing when $x(s) \in\left(\eta_{0}, \eta_{1}\right)$.

Secondly consider the inequality $F_{\alpha}[(1-\epsilon) \xi+\epsilon] \leq \xi$. We note that

$$
F_{\alpha}[(1-\epsilon) \xi+\epsilon]=\xi \Longleftrightarrow F_{\alpha}(\mu)=(\mu-\epsilon) /(1-\epsilon)
$$

where $\mu=(1-\epsilon) \xi+\epsilon$. Now this equation in turn is equivalent to

$$
\begin{aligned}
1-F_{\alpha}(\mu) & =1-(\mu-\epsilon) /(1-\epsilon) \\
& \Longleftrightarrow F_{\beta}(1-\mu)=(1-\mu) /(1-\epsilon) \Longleftrightarrow F_{\beta}(\omega)=\omega /(1-\epsilon)
\end{aligned}
$$

where $\omega=1-\mu$. Thus the new equation $F_{\beta}(\omega)=\omega /(1-\epsilon)$ has precisely the same form as the earlier equation $F_{\alpha}(\theta)=\theta /(1-\epsilon)$. By essentially repeating the earlier algebraic manipulations it follows that $x(s)$ always decreases when $x(s) \in\left(\xi_{1}, \xi_{0}\right)$. 
Remark 3 We make two comments on the statement of Theorem 2. In the first place, Theorem 2 does not require a random perturbation. In the second place, we considered referring to $\left[\xi_{1}(\epsilon), \xi_{0}(\epsilon)\right]$ and $\left[\eta_{0}(\epsilon), \eta_{1}(\epsilon)\right]$ as capture intervals and to $\left[0, \xi_{1}(\epsilon)\right]$ and $\left[\eta_{1}(\epsilon), 1\right]$ as absorbing intervals, but ultimately decided these names were not entirely appropriate. Although capture is initiated in $\left[\xi_{1}(\epsilon), \xi_{0}(\epsilon)\right]$ and $\left[\eta_{0}(\epsilon), \eta_{1}(\epsilon)\right]$ the population is not permanently captured in either interval. Ultimately we decided to use the term proliferation interval to describe $\left[\xi_{1}(\epsilon), \xi_{0}(\epsilon)\right]$ and $\left[\eta_{0}(\epsilon), \eta_{1}(\epsilon)\right]$ and the term capture interval to describe $\left[0, \xi_{1}(\epsilon)\right]$ and $\left[\eta_{1}(\epsilon), 1\right]$.

Theorem 3 Let $\boldsymbol{r}=\{r(t)\}_{t \in \mathbb{N}}$ be an outcome generated by a sequence of independent identically distributed random variables, defined for each set $\left\{t_{k}\right\}_{k=1}^{s} \subset \mathbb{N}$ of distinct times by the cumulative distribution functions $\mathcal{F}_{t_{1}, \ldots, t_{s}}\left(x_{1}, \ldots, x_{s}\right)=\prod_{j=1}^{s} \mathcal{F}\left(x_{j}\right)$, where $\mathcal{F}(x)$ is strictly increasing on $[0,1]$. If $2 \sqrt{\epsilon} /(1-\epsilon)<\alpha<(1-\epsilon) /(2 \sqrt{\epsilon})$ and $\left[\eta_{0}(\epsilon), \eta_{1}(\epsilon)\right]$ and $\left[\xi_{1}(\epsilon), \xi_{0}(\epsilon)\right]$ are the proliferation intervals defined in Theorem 2, then for each outcome $\boldsymbol{r}=\{r(t)\}_{t \in \mathbb{N}} \in[0,1]^{\infty}$ we have $\liminf _{t \rightarrow \infty} F_{\alpha, \epsilon, \boldsymbol{r}}^{(t)}(x)=$ $\eta_{1}(\epsilon)$ for all $x \in\left[\eta_{0}(\epsilon), 1\right]$, and $\limsup _{t \rightarrow \infty} F_{\alpha, \epsilon, r}^{(t)}(x)=\xi_{1}(\epsilon)$ for all $x \in\left[0, \xi_{0}(\epsilon)\right]$. More precisely, for each outcome $\boldsymbol{r} \in[0,1]^{\infty}$, there exists $p_{\boldsymbol{r}} \in\left(\xi_{0}(\epsilon), \eta_{0}(\epsilon)\right) \operatorname{such}$ that $\liminf _{t \rightarrow \infty} F_{\alpha, \epsilon, \boldsymbol{r}}^{(t)}(x)=\eta_{1}(\epsilon)$ for all $x \in\left(p_{\boldsymbol{r}}, 1\right]$, and $\lim \sup _{t \rightarrow \infty} F_{\alpha, \epsilon, \boldsymbol{r}}^{(t)}(x)=\xi_{1}(\epsilon)$ for all $x \in\left[0, p_{\boldsymbol{r}}\right)$. Furthermore, if $x(s) \in\left[\eta_{0}(\epsilon), \eta_{1}(\epsilon)\right]$ for some $s \in \mathbb{N}$, then $x(t) \geq \eta_{1}(\epsilon)$ after a finite number of additional iterations. Similarly, if $x(s) \in\left[\xi_{1}(\epsilon), \xi_{0}(\epsilon)\right]$ for some $s \in \mathbb{N}$, then $x(t) \leq \xi_{1}(\epsilon)$ after a finite number of additional iterations. Since $x(t) \in\left[\eta_{1}(\epsilon), 1\right] \Rightarrow x(t+1) \in\left[\eta_{1}(\epsilon), 1\right]$ and $x(t) \in\left[0, \xi_{1}(\epsilon)\right] \Rightarrow x(t+1) \in\left[0, \xi_{1}(\epsilon)\right]$ it follows that $\mathbb{P}\left[x(t) \in\left[\eta_{1}(\epsilon), 1\right] \cup\left[0, \xi_{1}(\epsilon)\right]\right]=1$.

Proof For convenience we will once again write $\xi_{j}=\xi_{j}(\epsilon)$ and $\eta_{j}=\eta_{j}(\epsilon)$ for each $j=0,1$. In proving Theorem 2 we showed that $x(s)$ always increases when $x(s) \in\left(\eta_{0}, \eta_{1}\right)$. In most cases the increase exceeds a well-defined minimum value. Suppose $x(s) \in\left[\eta_{0}, \eta_{1}\right]$ and $r(s)>\delta$. Since $F_{\alpha}(x)$ is monotone increasing, and $F_{\alpha}^{\prime}(x)$ is monotone decreasing for $x>p_{r}$, we deduce that

$$
\begin{aligned}
x(s+1) & =F_{\alpha}[(1-\epsilon) x(s)+\epsilon r(s)] \\
& \geq F_{\alpha}[(1-\epsilon) x(s)+\epsilon \delta] \\
& \geq F_{\alpha}[(1-\epsilon) x(s)]+F_{\alpha}^{\prime}[(1-\epsilon) x(s)+\epsilon \delta] \epsilon \delta .
\end{aligned}
$$

If $x(s+1)<\eta_{1}$, then we must have

$$
(1-\epsilon) x(s)+\epsilon \delta<(1-\epsilon) x(s)+\epsilon r(s)=F_{\alpha}^{-1}[x(s+1)]<F_{\alpha}^{-1}\left[\eta_{1}\right]=(1-\epsilon) \eta_{1}<\eta_{1} .
$$

Therefore $F_{\alpha}^{\prime}[(1-\epsilon) x(s)+\epsilon \delta] \geq F_{\alpha}^{\prime}\left(\eta_{1}\right)$. Finally we recall that $F_{\alpha}[(1-\epsilon) x(s)] \geq x(s)$. It follows that

$$
x(s+1) \geq x(s)+F_{\alpha}^{\prime}\left(\eta_{1}\right) \epsilon \delta=x(s)+c
$$

where $c=F_{\alpha}^{\prime}\left[\eta_{1}\right] \epsilon \delta=c(\delta)>0$. Therefore we always have either $x(s+1) \geq \eta_{1}$ or $x(s+1) \geq x(s)+c$ and $r(s)>\delta$. Since $\mathbb{P}[r(t)>\delta]=1-\mathcal{F}(\delta)>0$ we must have $x(t)>\eta_{1}$ after a finite number of generations. Thus $x(t) \in\left[\eta_{1}, 1\right]$ for some $t>s$. Since $r(t) \geq 0$ we have $(1-\epsilon) x(t)+\epsilon r(t) \geq(1-\epsilon) \eta_{1}$ and since $F_{\alpha}(x)$ is increasing it can be seen that

$$
x(t+1)=F_{\alpha}[(1-\epsilon) x(t)+\epsilon r(t)] \geq F_{\alpha}\left[(1-\epsilon) \eta_{1}\right]=\eta_{1} .
$$


Hence $x(t+1) \in\left[\eta_{1}, 1\right]$. It follows that $x(t) \in\left[\eta_{1}, 1\right]$ for all subsequent $t$. Therefore $\liminf _{t \rightarrow \infty} x(t) \geq$ $\eta_{1}$. We can improve this result. Suppose $\liminf _{t \rightarrow \infty} x(t)=\eta_{1}+\Delta \eta \in\left(\eta_{1}, 1\right]$. If we define $G(h)=$ $F_{\alpha}\left[(1-\epsilon)\left(\eta_{1}+\Delta \eta\right)+h\right]-\left[\left(\eta_{1}+\Delta \eta\right)-h\right]$ for $h \in\left[0,1-\left(\eta_{1}+\Delta \eta\right)\right]$ then

$$
F_{\alpha}\left[(1-\epsilon)\left(\eta_{1}+\Delta \eta\right)\right]<\eta_{1}+\Delta \eta \Longleftrightarrow G(0)<0 .
$$

Since $G(h)$ is right continuous at $h=0$ we can find $\gamma>0$ with

$$
G(\gamma)<0 \Longleftrightarrow F_{\alpha}\left[(1-\epsilon)\left(\eta_{1}+\Delta \eta\right)+\gamma\right]<\left[\eta_{1}+\Delta \eta-\gamma\right]
$$

Now our supposition means there is a strictly increasing sequence $\left\{t_{j}\right\}_{j \in \mathbb{N}} \subseteq \mathbb{N}$ with $x\left(t_{j}\right)<\eta_{1}+$ $\Delta \eta+\gamma$ for all $j \in \mathbb{N}$. It follows that for all $j \in \mathbb{N}$ with $r\left(t_{j}\right)<\gamma$ we have

$$
\begin{aligned}
x\left(t_{j}+1\right) & =F_{\alpha}\left[(1-\epsilon) x\left(t_{j}\right)+\epsilon r\left(t_{j}\right)\right] \\
& <F_{\alpha}\left[(1-\epsilon)\left(\eta_{1}+\Delta \eta+\gamma\right)+\epsilon \gamma\right] \\
& =F_{\alpha}\left[(1-\epsilon)\left(\eta_{1}+\Delta \eta\right)+\gamma\right] \\
& <\eta_{1}+\Delta \eta-\gamma .
\end{aligned}
$$

The elements in $\boldsymbol{r}$ are generated independently of $t \in \mathbb{N}$ and so $\mathbb{P}\left[r\left(t_{j}\right)<\gamma\right]=\lim _{x \uparrow \gamma} \mathcal{F}(\gamma)=\sigma>0$ is the same for all $j \in \mathbb{N}$. The probability that $r\left(t_{j}\right)<\gamma$ for some $j \leq m$ is given by

$$
\mathbb{P}\left[\exists m \in \mathbb{N} \text { such that } r\left(t_{j}\right)<\gamma \text { for some } j \leq m\right]=\sum_{j=0}^{m-1} \sigma(1-\sigma)^{j} \uparrow 1
$$

as $m \rightarrow \infty$. Thus we are certain to eventually find $t=s(1)$ with $x(t)<\eta_{1}+\Delta \eta+\gamma$ and $r(t)<\gamma$. Hence $x(t+1)<\eta_{1}+\Delta \eta-\gamma$. By repeating this argument it follows with equal certainty that we will find another value $t=s(2)>s(1)$ with $x(t+1)<\eta_{1}+\Delta \eta-\gamma$. By repeating this argument ad infinitum we can find a strictly increasing infinite sequence of integers $\{s(p)\}_{p \in \mathbb{N}}$ with $x(t+1)<$ $\eta_{1}+\Delta \eta-\gamma$ for all $t \in\{s(p)\}_{p \in \mathbb{N}}$. This contradicts our assumption that $\lim \inf _{t \rightarrow \infty} x(t)=\eta_{1}+\Delta \eta$. Thus the assumption is false and hence $\liminf _{t \rightarrow \infty} x(t)=\eta_{1}$.

In proving Theorem 2 we showed that the inequality $F_{\alpha}[(1-\epsilon) \xi+\epsilon] \leq \xi$ was simply an equivalent reformulation of the inequality $F_{\alpha}[(1-\epsilon) \eta] \geq \eta$. We already showed that if $x(s) \in\left(\eta_{0}, \eta_{1}\right)$ for some $s \in \mathbb{N}$ then $\lim \inf _{t \rightarrow \infty} x(t) \geq \eta_{1}$. By essentially repeating these arguments we can now show that if $x(s) \in\left(\xi_{1}, \xi_{0}\right)$ for some $s \in \mathbb{N}$ then $\lim _{\sup _{t \rightarrow \infty}} x(t) \leq \xi_{1}$. Once again we can do slightly better and prove that if $x(s) \in\left[0, \xi_{0}\right]$ for some $s \in \mathbb{N}$ then $\lim _{\sup _{t \rightarrow \infty}} x(t)=\xi_{1}$. The proof is similar to our earlier proof that if $x(s) \in\left[\eta_{0}, 1\right]$ for some $s \in \mathbb{N}$ then $\liminf \inf _{t \rightarrow \infty} x(t)=\eta_{1}$.

Finally we show that the quasi-limit function is an approximate step function. Let $\boldsymbol{r} \in[0,1]^{\infty}$ be an outcome generated by the sequence of independent identically distributed random variables. Choose $x \in\left(\xi_{0}, \eta_{0}\right)$ and let $x(1)=x$. Let us suppose that for some $\delta>0$ the sequence $\{x(t)\}_{t \in \mathbb{N}}$ never enters the region $\left[0, \xi_{0}+\delta\right)$. Define

$$
G(x, \mu)=F_{\alpha}[(1-\epsilon) x+\epsilon \mu]-x
$$


for $(x, \mu) \in[0,1] \times\left[\eta_{1}, 1\right]$. We have chosen $\xi_{0}$ so that $F\left[(1-\epsilon) \xi_{0}+\epsilon\right]=\xi_{0} \Longleftrightarrow G\left(\xi_{0}, 1\right)=0$ and $F[(1-\epsilon) x+\epsilon]>x \Longleftrightarrow G(x, 1)>0$ for $x \in\left(\xi_{0}, 1\right)$. Since $G(x, 1)$ is continuous on $\left(\xi_{0}, 1\right)$ it follows that for $x \in\left[\xi_{0}+\delta, \eta_{1}\right]$ we have $G(x, 1)>2 d$ for some $d=d(\delta)>0$. We also know that $G(x, \mu)$ is uniformly continuous on $\left[\xi_{0}+\delta, \eta_{1}\right] \times\left[\eta_{1}, 1\right]$. Therefore we can find $\mu_{0} \in\left[\eta_{1}, 1\right)$ such that

$$
G\left(x, \mu_{0}\right) \geq d \Longleftrightarrow F_{\alpha}\left[(1-\epsilon) x+\epsilon \mu_{0}\right] \geq x+d
$$

for all $x \in\left[\xi_{0}+\delta, \eta_{1}\right]$. If $x(s) \in\left[\xi_{0}+\delta, \eta_{1}\right]$ and $r(s)>\mu_{0}$ then $x(s+1)>x(s)+d$. If we have $k$ consecutive values $r(s)>\mu_{0}$ then $x(s+k)>x(s)+k d$. If we choose $k=k(\delta)=\left\lfloor\left(\eta_{1}-\xi_{0}-\delta\right) / d+1\right\rfloor$ and $x(s) \in\left[\xi_{0}+\delta, \eta_{1}\right]$ then it follows that $x(s+k)>\eta_{1}$. Since

$$
\mathbb{P}\left[r(t) \geq \mu_{0}\right]=1-\lim _{x \uparrow \mu_{0}} \mathcal{F}(x)=\tau>0
$$

for all $t \in \mathbb{N}$ it follows that for a batch of $k$ successive iterations

$$
\mathbb{P}\left[r(t) \geq \mu_{0} \text { for all } t=1,2, \ldots, k\right]=\tau^{k}>0
$$

and so the probability of one such batch in the first $m$ batches is

$$
\begin{array}{r}
\mathbb{P}\left[\exists m \in \mathbb{N} \text { and } s \in\{1, \ldots, m\} \text { with } r(t) \geq \mu_{0} \text { for } t\right. \\
=(s-1) k+1, \ldots, s k] \\
=\sum_{j=0}^{m-1} \tau^{k}\left(1-\tau^{k}\right)^{j} \uparrow 1
\end{array}
$$

as $m \rightarrow \infty$. Thus the desired sequence of $k$ successive times with $r(t) \geq \eta_{1}$ is certain to eventually occur. Therefore the sequence $\{x(t)\}_{t \in \mathbb{N}}$ will eventually be captured within the region $\left[\eta_{0}, 1\right]$ and consequently $\liminf \operatorname{in}_{t \rightarrow \infty} x(t)=\eta_{1}$.

Let us suppose that for some $\delta>0$ the sequence $\{x(t)\}_{t \in \mathbb{N}}$ never enters the region $\left(\eta_{0}-\delta, 1\right]$. A similar argument to the one used above now shows that if $x(s) \in\left(\xi_{1}, \xi_{0}\right)$ for some $s \in \mathbb{N}$ then it is certain to eventually be captured within the interval $\left[0, \xi_{1}\right]$ and consequently $\lim \sup _{t \rightarrow \infty} x(t)=\xi_{1}$.

If we consider two sequences $\boldsymbol{x}=\{x(t)\}_{t \in \mathbb{N}}$ and $\boldsymbol{x}^{*}=\left\{x^{*}(t)\right\}_{t \in \mathbb{N}}$ generated by the same sequence of outcomes $\boldsymbol{r}=\{r(t)\}_{t \in \mathbb{N}}$ with $x(1)<x^{*}(1)$ then $x(t)<x^{*}(t)$ for all $t \in \mathbb{N}$. That is $x(1)<x^{*}(1) \Rightarrow$ $\boldsymbol{x}<\boldsymbol{x}^{*}$. We deduce that if $\liminf _{t \rightarrow \infty} x(t)=\eta_{1}$ then we also have $\liminf _{t \rightarrow \infty} x^{*}(t)=\eta_{1}$. Similarly if $\lim \sup _{t \rightarrow \infty} x^{*}(t)=\xi_{1}$ then we also have $\lim \sup _{t \rightarrow \infty} x(t)=\xi_{1}$. We conclude that for each outcome $\boldsymbol{r} \in[0,1]^{\infty}$ there exists a unique point $p_{\boldsymbol{r}}$ such that $\liminf _{t \rightarrow \infty} x(t)=\eta_{1}$ when $x(1) \in\left(p_{\boldsymbol{r}}, 1\right]$ and $\lim \sup _{t \rightarrow \infty} x(t)=\xi_{1}$ when $x(1) \in\left[0, p_{\boldsymbol{r}}\right)$. Thus we have

$$
\liminf _{t \rightarrow \infty} F_{\alpha, \epsilon, \boldsymbol{r}}^{(t)}(x)=\eta_{1}(\epsilon)
$$

for $x \in\left(p_{\boldsymbol{r}}, 1\right]$ and

$$
\limsup _{t \rightarrow \infty} F_{\alpha, \epsilon, \boldsymbol{r}}^{(t)}(x)=\xi_{1}(\epsilon)
$$

for $x \in\left[0, p_{\boldsymbol{r}}\right)$. Note that (10) and (11) show that $\left[\eta_{0}(\epsilon), \eta_{1}(\epsilon)\right] \rightarrow\left(p^{*}, 1\right)$ where $p^{*}=1 /\left(1+\alpha^{2}\right)$ is the fixed point of the unperturbed mapping as $\epsilon \rightarrow 0$. Corresponding estimates can be derived to show that $\left[\xi_{1}(\epsilon), \xi_{0}(\epsilon)\right] \rightarrow\left(0, p^{*}\right)$ as $\epsilon \rightarrow 0$. 
Remark 4 In Theorem 3 we could use a uniform distribution with $\mathcal{F}(x)=x$ for all $x \in[0,1]$ or a binary distribution with $\mathcal{F}(x)=1 / 2$ for all $x \in[0,1)$. The perturbation in Theorem 2 may be deterministic or random. In the case of a random perturbation the generating distribution need not be stationary.

\section{Evolution of the general model}

We begin this section with general remarks about how we expect to generalize the ideas in Theorem 2 and Theorem 3. We then consider what happens for a particular case in $\mathcal{S}_{2}$ before moving on to some general results for $\mathcal{S}_{n-1}$. The basic idea is to look at a single component $p_{j}(t)$ and determine how that component will behave. In essence we argue that, provided the magnitude $\epsilon$ of the perturbation is sufficiently small, there are proliferation regions $\mathcal{D}_{j, \epsilon}$, near each stable vertex $V_{j}$, where the deterministic movement towards $V_{j}$ at each step outweighs the disruption caused by the random perturbation. If the proliferation region $\mathcal{D}_{j, \epsilon}$ contains a suitable $n-2$ dimensional polyhedral barrier set $\mathcal{P}_{j, \epsilon}=\left\{\boldsymbol{p} \in \mathcal{S}_{n-1} \mid p_{j}=\alpha_{j, \epsilon}\right\}$ for some $\alpha_{j, \epsilon} \in(0,1)$ which isolates the vertex $V_{j}$ from the other vertices of the simplex then we can define an associated capture region $\mathcal{C}_{j, \epsilon}=\left\{\boldsymbol{p} \in \mathcal{S}_{n-1} \mid p_{j} \geq \alpha_{j, \epsilon}\right\}$ with the property that $\boldsymbol{p}(s) \in \mathcal{C}_{j, \epsilon}$ implies $\boldsymbol{p}(s+1) \in \mathcal{C}_{j, \epsilon}$. When the population does not lie in any of these capture regions it is possible for the random perturbations to dominate the deterministic evolution. We argue that a sufficiently long string of successive random perturbations, with a high value of $r_{j}(t)$, will guarantee that the population enters the capture region $\mathcal{C}_{j, \epsilon}$ for $V_{j}$. From a probabilistic viewpoint this could be any one of the active capture regions. We will show that such a sequence must eventually occur.

Example 3 Let

$$
R=\left[\begin{array}{rrr}
8 & 1 & -1 \\
1 & 6 & -1 \\
-1 & -1 & 7
\end{array}\right]
$$

and define $W(c)=R+(c+1) \mathbf{1 1}^{T}$ where $c \geq 0$. The matrix $W=W(c)=\left[w_{i j}(c)\right]$ is symmetric and positive definite with $\left.\left.w_{i j}\right) c\right) \geq 0$ for all $i, j \in 1, \ldots, n$. Elementary arguments show that the unperturbed evolution defined by $W=W(c)$ is only weakly dependent on $c \geq 0$. Indeed it is easy to show that the fixed points do not depend on the value of $c$. The most significant difference is that the intergenerational step size decreases as $c$ increases. For $c=0$ and $c=10$ we have

$$
W(0)=\left[\begin{array}{lll}
9 & 2 & 0 \\
2 & 7 & 0 \\
0 & 0 & 8
\end{array}\right] \text { and } W(10)=\left[\begin{array}{lll}
19 & 12 & 10 \\
12 & 17 & 10 \\
10 & 10 & 18
\end{array}\right]
$$

Note that $W(10)$ is the same matrix used in Example 2. We are mainly interested in the case where the evolution is subject to random perturbations in the form

$$
\boldsymbol{p}(t+1)=(1-\epsilon)[\boldsymbol{p}(t) \circ \boldsymbol{w}(t)] / w(t)+\epsilon \boldsymbol{r}(t)
$$


for all $t \in \mathbb{N}$ where $\boldsymbol{r}=\{\boldsymbol{r}(t)\}_{t \in \mathbb{N}} \in \mathcal{S}_{2}{ }^{\infty}$ is an outcome generated by a sequence of independent identically distributed random vectors. When $c=0$ and $\epsilon=0.1$ and the perturbation is defined by a uniform distribution on $\mathcal{S}_{2}$ our numerical experiments suggest that $\boldsymbol{p}(t)$ is eventually captured in one of three regions in $\mathcal{S}_{2}$ near the stable fixed points for the unperturbed mapping at vertices $\boldsymbol{e}_{1}$, $\boldsymbol{e}_{2}$ and $\boldsymbol{e}_{3}$. When $c=10$ and $\epsilon=0.1$ the deterministic component of the step size is relatively much smaller than the random component and although the behaviour is generally similar our numerical experiments suggest that the evolution is no longer captured near the stable fixed point for the unperturbed mapping at vertex $\boldsymbol{e}_{2}$ but will eventually be captured near either $\boldsymbol{e}_{1}$ or $\boldsymbol{e}_{3}$. Some typical perturbed evolutionary paths are shown in Figure 6.
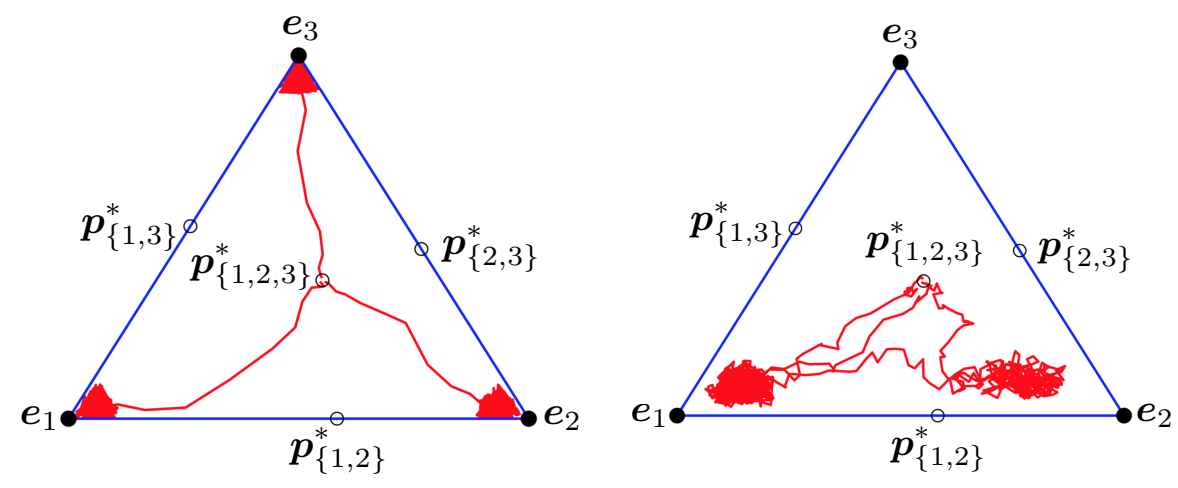

Figure 6: Evolutionary paths for the perturbed discrete-time model in Example 3 with $W=W(c)$ and $\epsilon=0.1$ starting at $\boldsymbol{p}_{\{1,2,3\}}^{*}$ and showing three different evolutionary paths over 1000 generations for $c=0$ (left) and $c=10$ (right). When $c=0$ the evolutionary paths are always captured near one of the vertices but when $c=10$ the graph on the right shows that one of the evolutionary paths approaches $\boldsymbol{e}_{2}$ but eventually escapes and is then apparently captured near $\boldsymbol{e}_{1}$.

\subsection{A general method for finding capture regions.}

If $W^{-1} \mathbf{1}>\mathbf{0}$ and $\epsilon=0$ then the minimum fitness occurs at the unstable fixed point $\boldsymbol{p}^{*}=$ $W^{-1} \mathbf{1} /\left(\mathbf{1}^{T} W^{-1} \mathbf{1}\right) \in \operatorname{int}\left(\mathcal{S}_{n-1}\right)$ and at least one vertex $\boldsymbol{e}_{j} \in \mathcal{S}_{n-1}$ for $j=1, \ldots, n$ will be a stable fixed point. For sufficiently small $\epsilon \in(0,1]$ we will show that in certain regions the deterministic component of the evolution dominates the random component and that evolutionary paths will always be captured near one or other of the vertices. As $\epsilon \in(0,1]$ increases the deterministic component becomes less dominant and the capture regions eventually disappear. Our basic idea is to find regions where the relative frequency of one particular genotype increases irrespective of the perturbation. Once again we consider a particular example. 
Example 4 Let

$$
W=\left[\begin{array}{ccc}
11 & 4 & 2 \\
4 & 9 & 2 \\
2 & 2 & 10
\end{array}\right]
$$

The vertices $V_{1}, V_{2}$ and $V_{3}$ of $\mathcal{S}_{2}$ are defined respectively by the vectors $\boldsymbol{e}_{1}, \boldsymbol{e}_{2}$ and $\boldsymbol{e}_{3}$. Consider the vertex $V_{1}$. In the case of the unperturbed mapping we wish to find a set in the form

$$
\mathcal{C}_{1,0}=\left\{\boldsymbol{p} \in \mathcal{S}_{2} \mid p_{1} \geq \alpha\right\}
$$

where $\alpha \in[0,1]$ such that each point $\boldsymbol{p} \in \mathcal{C}_{1,0}$ evolves at the next generation into a point $\boldsymbol{q}=$ $\boldsymbol{p} \circ(W \boldsymbol{p}) /\left(\boldsymbol{p}^{T} W \boldsymbol{p}\right) \in \mathcal{C}_{1,0}$. The evolution is completely described by writing

$$
\boldsymbol{p}_{\beta}(\alpha)=\left[\begin{array}{c}
p_{1, \beta}(\alpha) \\
p_{2, \beta}(\alpha) \\
p_{3, \beta}(\alpha)
\end{array}\right]=\left[\begin{array}{c}
\alpha \\
(1-\beta)(1-\alpha) \\
\beta(1-\alpha)
\end{array}\right] \in \mathcal{S}_{2}
$$

and

$$
\boldsymbol{q}_{\beta}(\alpha)=\frac{\boldsymbol{p}_{\beta}(\alpha) \circ\left[W \boldsymbol{p}_{\beta}(\alpha)\right]}{\boldsymbol{p}_{\beta}(\alpha)^{T} W \boldsymbol{p}_{\beta}(\alpha)} \in \mathcal{S}_{2}
$$

for all $\alpha, \beta \in[0,1]$. For each $\beta \in[0,1]$ we wish to find $\alpha$ so that $p_{1, \beta}(\alpha) \geq \alpha \Rightarrow q_{1, \beta}(\alpha) \geq \alpha$. In the first instance it is convenient to consider a slightly different problem. We will instead try to find $\alpha \in[0,1]$ so that $q_{1, \beta}(\alpha) \geq p_{1, \beta}(\alpha)=\alpha$. Thus we wish to define a suitable proliferation region

$$
\mathcal{D}_{1,0}=\left\{\boldsymbol{p}_{\beta}(\alpha) \in \mathcal{S}_{2} \mid q_{1, \beta}(\alpha) \geq p_{1, \beta}(\alpha)\right\} .
$$

For $\boldsymbol{p}_{\beta}(\alpha) \in \mathcal{D}_{1,0}$ we require

$$
f(\alpha, \beta) / g(\alpha, \beta) \geq \alpha
$$

where we have defined

$$
f(\alpha, \beta)=p_{1, \beta}(\alpha)\left[W \boldsymbol{p}_{\beta}(\alpha)\right]_{1}=[11 \alpha+4(1-\beta)(1-\alpha)+2 \beta(1-\alpha)] \alpha
$$

and

$$
\begin{aligned}
g(\alpha, \beta)= & \boldsymbol{p}_{\beta}(\alpha)^{T} W \boldsymbol{p}_{\beta}(\alpha) \\
= & 11 \alpha^{2}+8(1-\beta) \alpha(1-\alpha)+4 \beta \alpha(1-\alpha)+9(1-\beta)^{2}(1-\alpha)^{2} \\
& \quad+4 \beta(1-\beta)(1-\alpha)^{2}+10 \beta^{2}(1-\alpha)^{2} .
\end{aligned}
$$

We can simplify (12) to show that we require

$$
\alpha(\alpha-1)\left[\left(15 \beta^{2}-10 \beta+12\right) \alpha-\left(15 \beta^{2}-12 \beta+5\right)\right] \leq 0 .
$$

If $\alpha \in(0,1)$ we must have

$$
\left(15 \beta^{2}-12 \beta+5\right) /\left(15 \beta^{2}-10 \beta+12\right) \leq \alpha<1 .
$$


If $\beta=0$ the point $\boldsymbol{p}_{0}(\alpha)$ lies on the line $V_{1} V_{2}$ and the boundary of $\mathcal{D}_{1,0}$ is defined by $5 / 12 \leq \alpha<1$. If $\beta=1$ the point $\boldsymbol{p}_{1}(\alpha)$ lies on the line $V_{1} V_{3}$ and the boundary of $\mathcal{D}_{1,0}$ is defined by $8 / 17 \leq \alpha<1$. For $\beta \in(0,1)$ the boundary of $\mathcal{D}_{1,0}$ lies in the interior of $\mathcal{S}_{2}$ and is defined by the equation

$$
\alpha=\left(15 \beta^{2}-12 \beta+5\right) /\left(15 \beta^{2}-10 \beta+12\right) .
$$

We note in passing that the internal fixed point $\boldsymbol{p}_{\{1,2,3\}}^{*}=W^{-1} \mathbf{1} /\left(\mathbf{1}^{T} W^{-1} \mathbf{1}\right)$ lies on this interior boundary. The remaining boundary point is the vertex $V_{1}$ where $\alpha=1$ and $\boldsymbol{p}_{\beta}(1)=\boldsymbol{e}_{1}$. Because $q_{1, \beta}(\alpha)=p_{1, \beta}(\alpha)$ on the boundary of $\mathcal{D}_{1,0}$ it is clear that the fixed points $\boldsymbol{p}_{\{1,2\}}^{*}=\boldsymbol{p}_{0}(5 / 12)$ and $\boldsymbol{p}_{\{1,3\}}^{*}=\boldsymbol{p}_{1}(8 / 17)$ also lie on the boundary.

Now consider the perturbed mapping. We use $\boldsymbol{p}_{\beta}(\alpha)$ and $\boldsymbol{q}_{\beta}(\alpha)$ as before and define the perturbed image by setting $\boldsymbol{q}_{\beta, \epsilon, \boldsymbol{r}}(\alpha)=(1-\epsilon) \boldsymbol{q}_{\beta}(\alpha)+\epsilon \boldsymbol{r}$ where $\boldsymbol{r} \in \mathcal{S}_{2}$ is chosen randomly. For $\epsilon \in(0,1]$ and sufficiently small we wish to find $\left[\alpha_{1}(\epsilon), \alpha_{2}(\epsilon)\right] \subset(0,1)$ such that for all $\alpha \in\left[\alpha_{1}(\epsilon), \alpha_{2}(\epsilon)\right]$ we have $q_{1, \beta, \epsilon, \boldsymbol{r}}(\alpha) \geq p_{1, \beta}(\alpha)=\alpha$ irrespective of the perturbation $\boldsymbol{r} \in \mathcal{S}_{2}$. We define an associated proliferation region

$$
\mathcal{D}_{1, \epsilon}=\left\{\boldsymbol{p}_{\beta}(\alpha) \in \mathcal{S}_{2} \mid q_{1, \beta, \epsilon, \boldsymbol{r}}(\alpha) \geq p_{1, \beta}(\alpha)\right\} .
$$

Since we are considering only the first component the most difficult evolutions to capture are those with $r_{1}=\boldsymbol{e}_{1}^{T} \boldsymbol{r}=0$. Thus we require $\alpha=\alpha(\epsilon)$ such that

$$
(1-\epsilon) f(\alpha, \beta) / g(\alpha, \beta) \geq \alpha
$$

where $f(\alpha, \beta)$ and $g(\alpha, \beta)$ are the functions defined in (13) and (14) respectively. We can simplify (15) to give

$$
\alpha\left\{f_{1}(\beta) \alpha^{2}+g_{1}(\beta, \epsilon) \alpha+h_{1}(\beta, \epsilon)\right\} \leq 0
$$

where we have defined

$$
\begin{array}{r}
f_{1}(\beta)=15 \beta^{2}-10 \beta+12, \quad g_{1}(\beta, \epsilon)=-30 \beta^{2}+(22+2 \epsilon) \beta-(17-7 \epsilon) \\
\text { and } \quad h_{1}(\beta, \epsilon)=15 \beta^{2}-(12+2 \epsilon) \beta+(5+4 \epsilon) .
\end{array}
$$

The discriminant $\Delta_{1}=\Delta_{1}(\beta, \epsilon)$ for the quadratic equation

$$
f_{1}(\beta) \alpha^{2}+g_{1}(\beta, \epsilon) \alpha+h_{1}(\beta, \epsilon)=0
$$

is given by

$$
\begin{aligned}
\Delta_{1} & =g_{1}(\beta, \epsilon)^{2}-4 f_{1}(\beta) h_{1}(\beta, \epsilon) \\
& =\left(4 \epsilon^{2}-652 \epsilon+4\right) \beta^{2}+\left(28 \epsilon^{2}+496 \epsilon+28\right) \beta+\left(49 \epsilon^{2}-430 \epsilon+49\right) .
\end{aligned}
$$

If $\alpha \in(0,1]$ we wish to find values of $\beta \in[0,1]$ and $\epsilon \in[0,1]$ such that $\Delta_{1} \geq 0$. If we define $f_{2}(\epsilon)=4 \epsilon^{2}-652 \epsilon+4, g_{2}(\epsilon)=28 \epsilon^{2}+496 \epsilon+28$ and $h_{2}(\epsilon)=49 \epsilon^{2}-430 \epsilon+49$ then we can write the quadratic equation $\Delta_{1}=0$ in the form

$$
f_{2}(\epsilon) \beta^{2}+g_{2}(\epsilon) \beta+h_{2}(\epsilon)=0
$$


in which case the discriminant $\Delta_{2}=\Delta_{2}(\epsilon)$ is given by

$$
\Delta_{2}=g_{2}(\epsilon)^{2}-4 f_{2}(\epsilon) h_{2}(\epsilon)=176 \epsilon\left[923 \epsilon^{2}-4974 \epsilon+923\right] .
$$

It follows that $\Delta_{2} \geq 0$ when

$$
0 \leq \epsilon \leq 4974 / 1846-\sqrt{(4974 / 1846)^{2}-1}=\epsilon_{\max } \approx 0.1924
$$

Hence there are two real solutions $\beta_{1}=\beta_{1}(\epsilon)$ and $\beta_{2}=\beta_{2}(\epsilon)$ to (17) given by

$$
\begin{aligned}
\beta_{1}=-\frac{g_{2}(\epsilon)}{2 f_{2}(\epsilon)}-\sqrt{\left(\frac{g_{2}(\epsilon)}{2 f_{2}(\epsilon)}\right)^{2}-\frac{h_{2}(\epsilon)}{f_{2}(\epsilon)}}, \\
\beta_{2}=-\frac{g_{2}(\epsilon)}{2 f_{2}(\epsilon)}+\sqrt{\left(\frac{g_{2}(\epsilon)}{2 f_{2}(\epsilon)}\right)^{2}-\frac{h_{2}(\epsilon)}{f_{2}(\epsilon)}}
\end{aligned}
$$

for $\epsilon \in\left(0, \epsilon_{\max }\right)$. To be more precise in our analysis we need to consider several different cases. We will assume that $\epsilon \in\left[0, \epsilon_{\max }\right]$.

Case 1: In the first case suppose $f_{2}(\epsilon)>0$. Now we have

$$
f_{2}(\epsilon)>0 \Longleftrightarrow \epsilon^{2}-163 \epsilon+1>0 \Longleftrightarrow 0 \leq \epsilon<\epsilon_{1}
$$

where

$$
\epsilon_{1}=163 / 2-\sqrt{(163 / 2)^{2}-1} \approx 0.0061352 .
$$

Since $f_{2}(\epsilon), g_{2}(\epsilon)$ and $h_{2}(\epsilon)$ are all positive for $\epsilon \in\left(0, \epsilon_{1}\right)$ we have $\beta_{1}<\beta_{2}<0$. This means that for $\epsilon \in\left[0, \epsilon_{1}\right]$ we have $\Delta_{1}>0$ for all $\beta \in[0,1]$. Thus we have two real solutions $\alpha_{1}=\alpha_{1}(\beta, \epsilon)$ and $\alpha_{2}=\alpha_{2}(\beta, \epsilon)$ to $(16)$ given by

$$
\begin{aligned}
\alpha_{1}=-\frac{g_{1}(\beta, \epsilon)}{2 f_{1}(\beta, \epsilon)}-\sqrt{\left(\frac{g_{1}(\beta, \epsilon)}{2 f_{1}(\beta, \epsilon)}\right)^{2}-\frac{h_{1}(\beta, \epsilon)}{f_{1}(\beta, \epsilon)}} \\
\alpha_{2}=-\frac{g_{1}(\beta, \epsilon)}{2 f_{1}(\beta, \epsilon)}+\sqrt{\left(\frac{g_{1}(\beta, \epsilon)}{2 f_{1}(\beta, \epsilon)}\right)^{2}-\frac{h_{1}(\beta, \epsilon)}{f_{1}(\beta, \epsilon)}}
\end{aligned}
$$

for all $\beta \in[0,1]$.

Case 2a: Now suppose that $f_{2}(\epsilon)<0$. Therefore $\epsilon_{1}<\epsilon<\epsilon_{\max }$. In this case we know that $\Delta_{1}(\beta, \epsilon)=0$ has two solutions $\beta_{1}(\epsilon)$ and $\beta_{2}(\epsilon)$ and that $\Delta_{1}(\beta, \epsilon)>0$ for $\beta \in\left(\beta_{1}, \beta_{2}\right)$. We wish to find conditions that ensure $[0,1] \subseteq\left[\beta_{1}, \beta_{2}\right]$. Thus we require $\Delta_{1}(0, \epsilon)=49 \epsilon^{2}-430 \epsilon+49 \geq 0$ and $\Delta_{1}(1, \epsilon)=81 \epsilon^{2}-586 \epsilon+81 \geq 0$. The first condition gives

$$
\epsilon \leq \frac{215}{49}-\sqrt{\left(\frac{215}{49}\right)^{2}-1} \approx 0.1155 \quad \text { or } \quad \epsilon \geq \frac{215}{49}+\sqrt{\left(\frac{215}{49}\right)^{2}-1} \approx 8.6600
$$


while the second condition gives

$$
\epsilon \leq \frac{293}{81}-\sqrt{\left(\frac{293}{81}\right)^{2}-1} \approx 0.1410 \quad \text { or } \quad \epsilon \geq \frac{293}{81}+\sqrt{\left(\frac{293}{81}\right)^{2}-1} \approx 7.0936 .
$$

Thus we define

$$
\epsilon_{2}=\frac{215}{49}-\sqrt{\left(\frac{215}{49}\right)^{2}-1} \approx 0.1155
$$

and conclude that for $\epsilon_{1}<\epsilon<\epsilon_{2}$ we have $\Delta_{1}(0, \epsilon) \geq 0$ and $\Delta_{1}(1, \epsilon) \geq 0$ and hence $[0,1] \subseteq\left[\beta_{1}, \beta_{2}\right]$. Therefore the equation (16) has two real solutions for all $\beta \in[0,1]$.

Case 2b: Now define

$$
\epsilon_{3}=\frac{293}{81}-\sqrt{\left(\frac{293}{81}\right)^{2}-1} \approx 0.1410 .
$$

For $\epsilon_{2}<\epsilon<\epsilon_{3}$ we have $\Delta_{1}(0, \epsilon)<0$ and $\Delta_{1}(1, \epsilon)>0$. Thus we have $0 \leq \beta_{1}<1<\beta_{2}$ and there are two real solutions to (16) for all $\beta \in\left(\beta_{1}, 1\right)$.

Case 2c: For $\epsilon_{3}<\epsilon<\epsilon_{\max }$ we have $\Delta_{1}(0, \epsilon)<0$ and $\Delta_{1}(1, \epsilon)<0$. Thus we have $0 \leq \beta_{1}<\beta_{2}<1$ and there are two real solutions to (16) for all $\beta \in\left(\beta_{1}, \beta_{2}\right)$.

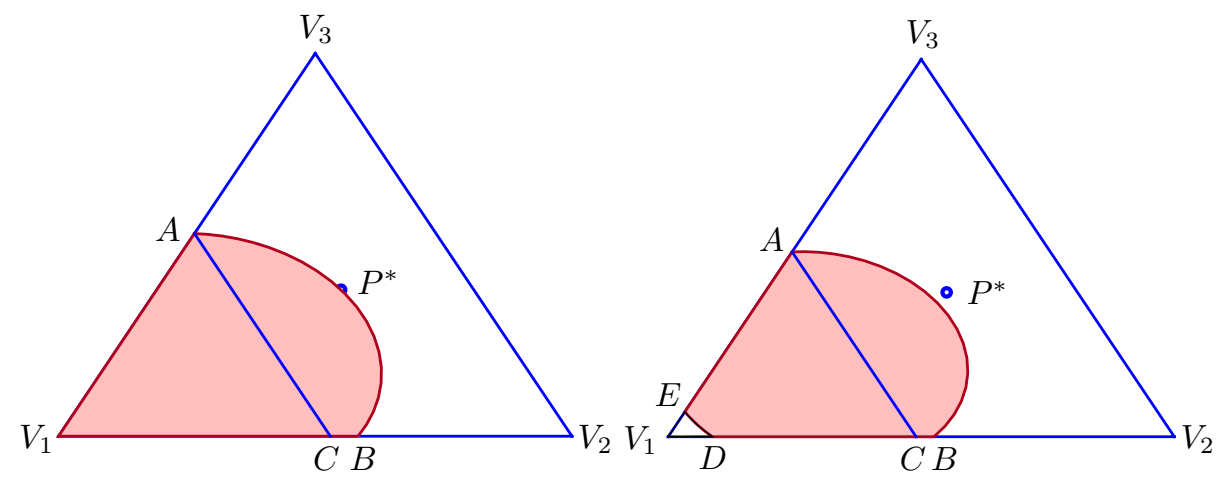

Figure 7: The vertices of the simplex are $V_{1}=e_{1}, V_{2}=e_{2}$ and $V_{3}=e_{3}$. The shaded regions $\mathcal{D}_{1, \epsilon}=A B C V_{1}$ (left) and $\mathcal{D}_{1, \epsilon}=A B C D E$ (right) are the sets where the relative frequency of genotype $\mathcal{G}_{1}$ increases in Example 4 with $\epsilon=0$ (left) and $\epsilon=0.05$ (right). The capture region for genotype $\mathcal{G}_{1}$ is $\mathcal{C}_{1, \epsilon}=A C V_{1}$ in each case. The point $P^{*}$ is the unstable fixed point for the unperturbed evolution.

We have shown that for each $\epsilon \in\left[0, \epsilon_{\max }\right]$ there are real numbers $\beta_{1}=\beta_{1}(\epsilon)$ and $\beta_{2}=\beta_{2}(\epsilon)$ with $\beta_{1} \leq \beta_{2}$ and real numbers $\alpha_{1}=\alpha_{1}(\beta, \epsilon)$ and $\alpha_{2}=\alpha_{2}(\beta, \epsilon)$ with $0 \leq \alpha_{1} \leq \alpha_{2} \leq 1$ for each $\beta \in\left(\beta_{1}, \beta_{2}\right)$ such that $p_{1, \beta}(\alpha)=\alpha$ implies $q_{1, \beta, \epsilon, r}(\alpha) \geq \alpha$ for $\alpha \in\left[\alpha_{1}(\beta, \epsilon), \alpha_{2}(\beta, \epsilon)\right]$ and $\beta \in\left[\beta_{1}(\epsilon), \beta_{2}(\epsilon)\right]$. Because each set

$$
\mathcal{D}_{1, \beta, \epsilon}=\left\{\boldsymbol{p}_{\beta}(\alpha) \in \mathcal{S}_{2} \mid \alpha \in\left[\alpha_{1}(\beta, \epsilon), \alpha_{2}(\beta, \epsilon)\right]\right\}
$$




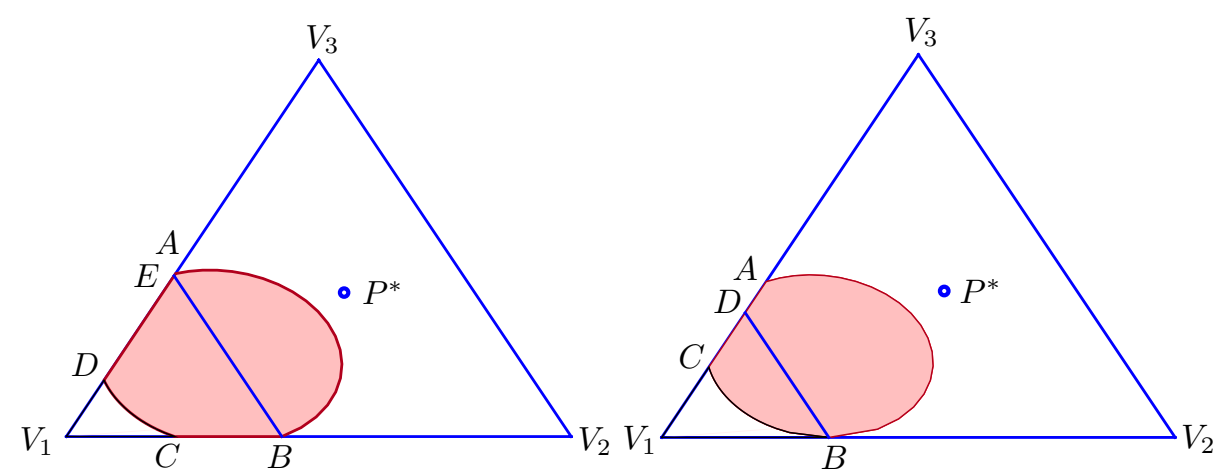

Figure 8: The vertices of the simplex are $V_{1}=\boldsymbol{e}_{1}, V_{2}=\boldsymbol{e}_{2}$ and $V_{3}=\boldsymbol{e}_{3}$. The shaded regions $\mathcal{D}_{1, \epsilon}=A B C D$ (left) and $\mathcal{D}_{1, \epsilon}=A B C$ (right) are the sets where the relative frequency of genotype $\mathcal{G}_{1}$ increases in Example 4 with $\epsilon=0.1$ (left) and $\epsilon=0.1155 \approx \epsilon_{2}$ (right). The capture regions for genotype $\mathcal{G}_{1}$ are $\mathcal{C}_{1, \epsilon}=E B V_{1}$ (left) and $\mathcal{C}_{1, \epsilon}=D B V_{1}$ (right). The point $P^{*}$ is the unstable fixed point for the unperturbed evolution.

is a straight line segment in $\mathcal{S}_{2}$ from $\alpha=\alpha_{1}(\beta, \epsilon)$ to $\alpha=\alpha_{2}(\beta, \epsilon)$ for the given value of $\beta \in$ $\left(\beta_{1}(\epsilon), \beta_{2}(\epsilon)\right)$ we can see that

$$
\mathcal{D}_{1, \epsilon}=\left\{\boldsymbol{p}_{\beta}(\alpha) \in \mathcal{S}_{2} \mid \beta \in\left[\beta_{1}(\epsilon), \beta_{2}(\epsilon)\right] \text { and } \alpha \in\left[\alpha_{1}(\beta, \epsilon), \alpha_{2}(\beta, \epsilon)\right]\right\} .
$$

We will show later that the proliferation set $\mathcal{D}_{1, \epsilon}$ is a closed convex set. In Figures 7 and 8 the curve $A B$ is the boundary of the set $\mathcal{D}_{1, \epsilon}$ where $\alpha=\alpha_{1}(\beta, \epsilon)$. The other boundary of $\mathcal{D}_{1, \epsilon}$ within $\mathcal{S}_{2}$ is where $\alpha=\alpha_{2}(\beta, \epsilon)$. In Figure 7 this boundary degenerates to the single point $V_{1}$ when $\epsilon=0$ and is shown as the curve $D E$ when $\epsilon=0.05$. In Figure 8 this boundary is shown as the curve $C D$ when $\epsilon=0.1$ and as the curve $B C$ when $\epsilon=0.1155 \approx \epsilon_{2}$. If $0 \leq \epsilon \leq \epsilon_{2}$ and we set

$$
\alpha_{1, \epsilon}=\max _{\beta \in[0,1]} \alpha_{1}(\beta, \epsilon)
$$

then the set

$$
\mathcal{C}_{1, \epsilon}=\left\{\boldsymbol{p} \in \mathcal{S}_{2} \mid \alpha \geq \alpha_{1, \epsilon}\right\}
$$

has the property that $\boldsymbol{p} \in \mathcal{C}_{1, \epsilon} \Longrightarrow \boldsymbol{q}=(1-\epsilon)[W \boldsymbol{p}] \circ \boldsymbol{p} /\left(\boldsymbol{p}^{T} W \boldsymbol{p}\right) \in \mathcal{C}_{1, \epsilon}$. In the language of discrete dynamical systems the region $\mathcal{C}_{1, \epsilon}$ can be thought of as a basin of attraction for the genotype $\mathcal{G}_{1}$ near the locally stable fixed point $\boldsymbol{e}_{1}$. We shall refer to $\mathcal{C}_{1, \epsilon}$ as a capture set for the genotype $\mathcal{G}_{1}$. In Figure 7 the set $\mathcal{C}_{1, \epsilon}$ is shown in both cases as the triangle $A C V_{1}$ and in Figure 8 it is shown as the triangle $E B V_{1}$ when $\epsilon=0.1$ and as the triangle $D B V_{1}$ when $\epsilon=0.1155 \approx \epsilon_{2}$.

The set $\mathcal{D}_{1, \epsilon}$ in which the relative frequency of genotype $\mathcal{G}_{1}$ increases no matter what random perturbation occurs is shown as the shaded region in Figures 7 to 10 for various values of $\epsilon$. In Figures 7 and 8 we have $0 \leq \epsilon \leq \epsilon_{2} \approx 0.1155$ and the set $\mathcal{D}_{1, \epsilon}$ is bounded in part by the edges $V_{1} V_{2}$ and $V_{1} V_{3}$ of the simplex $\mathcal{S}_{2}$ and hence separates the vertex $V_{1}$ from the other two vertices $V_{2}$ 


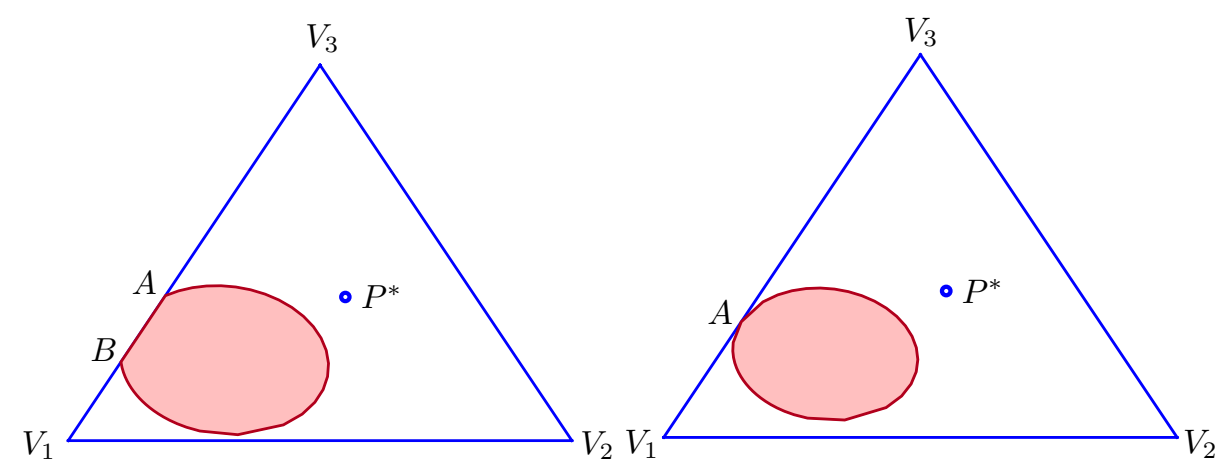

Figure 9: The vertices of the simplex are $V_{1}=\boldsymbol{e}_{1}, V_{2}=\boldsymbol{e}_{2}$ and $V_{3}=\boldsymbol{e}_{3}$. The shaded regions $\mathcal{D}_{1, \epsilon}=A B A$ (left) and $\mathcal{D}_{1, \epsilon}=A A$ (right) are the sets where the relative frequency of genotype $\mathcal{G}_{1}$ increases in Example 4 with $\epsilon=0.125$ (left) and $\epsilon=0.1385 \approx \epsilon_{3}$ (right). There is no (local) capture region for genotype $\mathcal{G}_{1}$ in either case.

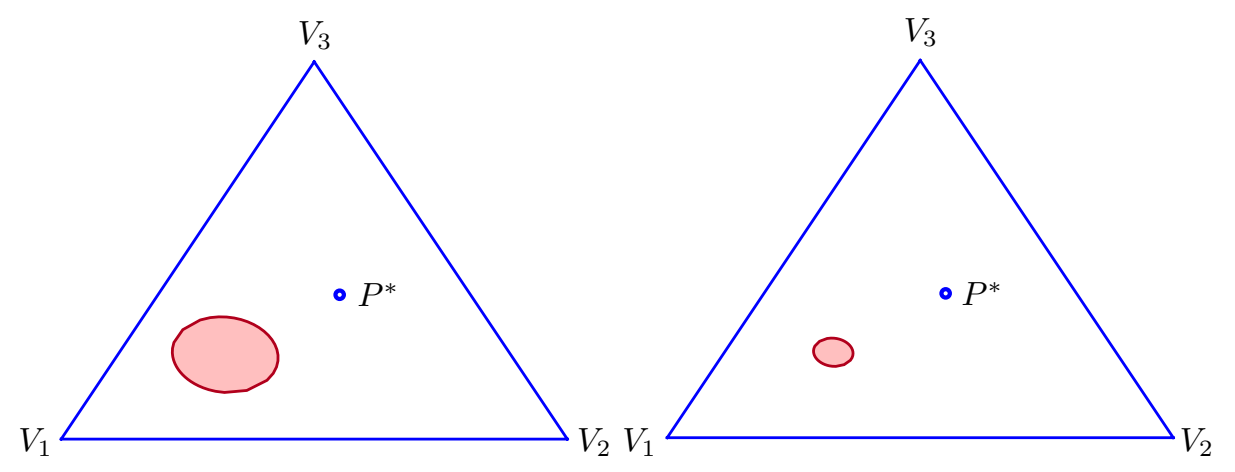

Figure 10: The vertices of the simplex are $V_{1}=\boldsymbol{e}_{1}, V_{2}=\boldsymbol{e}_{2}$ and $V_{3}=\boldsymbol{e}_{3}$. The shaded regions $\mathcal{D}_{1, \epsilon}$ are the sets where the relative frequency of genotype $\mathcal{G}_{1}$ increases in Example 4 with $\epsilon=0.175$ (left) and $\epsilon=0.19$ (right). There is no (local) capture region for genotype $\mathcal{G}_{1}$ in either case.

and $V_{3}$. In Figure 7 any trajectory that enters the capture region $\mathcal{C}_{1, \epsilon}=A C V_{1}$ for genotype $\mathcal{G}_{1}$, when $\epsilon=0$ (left) or $\epsilon=0.05$ (right), can never escape. In Figure 8 any trajectory that enters the capture region $\mathcal{C}_{1, \epsilon}=E B V_{1}$ for genotype $\mathcal{G}_{1}$ when $\epsilon=0.1$ (left), or the capture region $\mathcal{C}_{1, \epsilon}=D B V_{1}$ for genotype $\mathcal{G}_{1}$ when $\epsilon=0.1155 \approx \epsilon_{2}$ (right), can never escape. In Figures 9 and 10 we have $\epsilon_{2} \approx 0.1155<\epsilon<0.1924 \approx \epsilon_{\max }$ and there is no (local) capture region for genotype $\mathcal{G}_{1}$. In this example there are similar regions with similar behaviour for genotypes $\mathcal{G}_{2}$ and $\mathcal{G}_{3}$.

Remark 5 We wish to clarify our discussion of capture regions. The vector $\boldsymbol{p}(t)$ always lies in $\mathcal{S}_{n-1}$. As such $\mathcal{S}_{n-1}$ is technically a global capture region. We should really refer to the capture regions in our discussion as local capture regions, but for the sake of brevity, except where specific clarification is warranted, we refer to them simply as capture regions. The primary mechanism for capture of the population near a stable vertex $V_{j}$ is an unavoidable increase in the relative frequency of geneotype 
$\mathcal{G}_{j}$. This will happen if the random perturbation is sufficiently small and $\boldsymbol{p}(t)$ lies within a special proliferation region $\mathcal{D}_{j, \epsilon}$ where the deterministic trend outweighs the random perturbation. This region is formed by the intersection of an elliptical set $\mathcal{E}_{j, \epsilon}$, which we introduce in Section 6.2, and the simplex $\mathcal{S}_{n-1}$. Within $\mathcal{D}_{j, \epsilon}$ the relative frequency $p_{j}(t)$ will increase from one generation to the next irrespective of the perturbation. For $\epsilon>0$ the set $\mathcal{D}_{j, \epsilon}$ does not include the vertex $V_{j}$. See, for instance the shaded regions in Figure 8. It is technically possible that two different regions $\mathcal{D}_{j, \epsilon}$ and $\mathcal{D}_{k, \epsilon}$ will overlap and for that reason the guaranteed capture set for $\mathcal{G}_{j}$ is a slightly smaller polyhedral subset of $\mathcal{D}_{j, \epsilon}$. See the regions $V_{1} E B$ and $V_{1} D B$ in Figure 8. These are particular instances of the general regions $\mathcal{C}_{j, \epsilon}$ introduced in Section 6.2. The capture region $\mathcal{C}_{j, \epsilon}$ always includes the relevant vertex $V_{j}$. We note that when $\boldsymbol{p}(t)$ is very close to $V_{j}$ the random perturbation may override the deterministic trend, in which case $\boldsymbol{p}(t)$ may move back towards the proliferation region. However it can never escape from the capture set. As $\epsilon$ increases, the sets $\mathcal{D}_{j, \epsilon}$ shrink to the point where they can no longer be used to define capture regions. See Figures 9 and 10. The capture regions do not all disappear at the same value of $\epsilon$. If capture regions exist for a particular value of $\epsilon$ we will prove that the population is captured eventually with probability one.

\subsection{A general result about capture regions.}

Let $W=\left[w_{i j}\right] \in \mathbb{R}^{n \times n}$ be symmetric and positive definite with $w_{i j} \geq 0$ and assume $W^{-1} \mathbf{1}>\mathbf{0}$. Choose $\epsilon \in(0,1)$ and fix $j \in\{1,2, \ldots, n\}$. Consider the proliferation region $\mathcal{D}_{j}=\mathcal{D}_{j, \epsilon}$ defined by $\boldsymbol{p} \in \mathcal{S}_{n-1}$ and

$$
(1-\epsilon) q_{j} \geq p_{j} \Longleftrightarrow(1-\epsilon) \frac{p_{j}(W \boldsymbol{p})_{j}}{\boldsymbol{p}^{T} W \boldsymbol{p}} \geq p_{j}
$$

If $p_{j}>0$ we cancel by $p_{j}$ and multiply through by $\boldsymbol{p}^{T} W \boldsymbol{p}$ to show that (18) implies

$$
(1-\epsilon) \boldsymbol{e}_{j}^{T} W \boldsymbol{p} \geq \boldsymbol{p}^{T} W \boldsymbol{p} .
$$

We now consider a somewhat larger set $\mathcal{E}_{j}=\mathcal{E}_{j, \epsilon}$ defined by

$$
\mathcal{E}_{j}=\left\{\boldsymbol{p} \in \mathcal{H}_{n-1} \mid(1-\epsilon) \boldsymbol{e}_{j}^{T} W \boldsymbol{p} \geq \boldsymbol{p}^{T} W \boldsymbol{p}\right\}
$$

where $\mathcal{H}_{n-1} \subset \mathbb{R}^{n}$ is the hyperplane defined by $\boldsymbol{p}^{T} \mathbf{1}=1$ for all $\boldsymbol{p} \in \mathbb{R}^{n}$. Write $\boldsymbol{p}=\boldsymbol{p}^{*}+\boldsymbol{u}$ and $\boldsymbol{e}_{j}=\boldsymbol{p}^{*}+\boldsymbol{f}_{j}$ where $\boldsymbol{p}^{*}=W^{-1} \mathbf{1} /\left(\mathbf{1}^{T} W^{-1} \mathbf{1}\right)$ is the unstable fixed point in the interior of $\mathcal{S}_{n-1}$ and $\boldsymbol{u}^{T} \mathbf{1}=\boldsymbol{f}_{j}{ }^{T} \mathbf{1}=0$. Now we can write (19) in the form

$$
\begin{aligned}
(1-\epsilon)\left(\boldsymbol{p}^{*}+\boldsymbol{f}_{j}\right)^{T} W & \left(\boldsymbol{p}^{*}+\boldsymbol{u}\right) \geq\left(\boldsymbol{p}^{*}+\boldsymbol{u}\right)^{T} W\left(\boldsymbol{p}^{*}+\boldsymbol{u}\right) \\
& \Longleftrightarrow \quad-\epsilon /\left(\mathbf{1}^{T} W^{-1} \mathbf{1}\right)+(1-\epsilon) \boldsymbol{f}_{j}^{T} W \boldsymbol{u} \geq \boldsymbol{u}^{T} W \boldsymbol{u}
\end{aligned}
$$

because we have $\boldsymbol{p}^{* T} W \boldsymbol{p}^{*}=1 /\left(\mathbf{1}^{T} W^{-1} \mathbf{1}\right), \boldsymbol{u}^{T} W \boldsymbol{p}^{*}=\boldsymbol{u}^{T} \mathbf{1} /\left(\mathbf{1}^{T} W^{-1} \mathbf{1}\right)=0$ and $\boldsymbol{f}_{j}{ }^{T} W \boldsymbol{p}^{*}=\boldsymbol{f}_{j}{ }^{T} \mathbf{1} /\left(\mathbf{1}^{T} W^{-1} \mathbf{1}\right)=$ 0 . If we define the orthogonal projection matrix $P=I-\mathbf{1 1}^{T} / n$ then $P^{T}=P$ and we also have 
$\boldsymbol{u}=P \boldsymbol{u}$ and $\boldsymbol{f}_{j}=P \boldsymbol{f}_{j}$. Therefore

$$
\begin{aligned}
&-\epsilon /\left(\mathbf{1}^{T} W^{-1} \mathbf{1}\right)+(1-\epsilon) \boldsymbol{f}_{j}{ }^{T} W \boldsymbol{u} \geq \boldsymbol{u}^{T} W \boldsymbol{u} \\
& \Longleftrightarrow-\epsilon /\left(\mathbf{1}^{T} W^{-1} \mathbf{1}\right)+(1-\epsilon) \boldsymbol{f}_{j}{ }^{T} P^{T} W P \boldsymbol{u} \geq \boldsymbol{u}^{T} P^{T} W P \boldsymbol{u} \\
& \Longleftrightarrow-\epsilon /\left(\mathbf{1}^{T} W^{-1} \mathbf{1}\right)+(1-\epsilon) \boldsymbol{f}_{j}{ }^{T} X \boldsymbol{u} \geq \boldsymbol{u}^{T} X \boldsymbol{u}
\end{aligned}
$$

where we have defined $X=P^{T} W P$. Since $X \boldsymbol{u}=W \boldsymbol{u}$ when $\boldsymbol{u} \in \mathcal{S}_{n-1}$ but $X \mathbf{1}=\mathbf{0}$ it follows that $X$ is positive semi-definite with eigenvalues $\lambda_{1} \geq \cdots \geq \lambda_{n-1}>\lambda_{n}=0$ and there exists an orthogonal matrix $Q$ such that $Q^{T} X Q=\Lambda$ where $\Lambda=\operatorname{diag}\left(\lambda_{j}\right)$ is the diagonal matrix of eigenvalues. Therefore

$$
\begin{aligned}
& -\epsilon \kappa+(1-\epsilon) \boldsymbol{f}_{j}{ }^{T} X \boldsymbol{u} \geq \boldsymbol{u}^{T} X \boldsymbol{u} \\
& \quad \Longleftrightarrow \quad-\epsilon \kappa+(1-\epsilon)\left(Q^{T} \boldsymbol{f}_{j}\right)^{T}\left(Q^{T} X Q\right)\left(Q^{T} \boldsymbol{u}\right) \geq\left(Q^{T} \boldsymbol{u}\right)^{T}\left(Q^{T} X Q\right)\left(Q^{T} \boldsymbol{u}\right) \\
& \quad \Longleftrightarrow \quad-\epsilon \kappa+(1-\epsilon) \boldsymbol{g}_{j}^{T} \Lambda \boldsymbol{v} \geq \boldsymbol{v}^{T} \Lambda \boldsymbol{v}
\end{aligned}
$$

where $\boldsymbol{g}_{j}=Q^{T} \boldsymbol{f}_{j}$ and $\boldsymbol{v}=Q^{T} \boldsymbol{u}$ and we have defined $\kappa=1 /\left(\mathbf{1}^{T} W^{-1} \mathbf{1}\right)$. Now (23) can be rewritten as

$$
\lambda_{1} v_{1}^{2}+\cdots+\lambda_{n-1} v_{n-1}^{2} \leq(1-\epsilon)\left[\lambda_{1} g_{j, 1} v_{1}+\cdots+\lambda_{n-1} g_{j, n-1} v_{n-1}\right]-\epsilon \kappa
$$

or equivalently as

$$
\lambda_{1}\left(v_{1}-\delta_{1}\right)^{2}+\cdots+\lambda_{n-1}\left(v_{n-1}-\delta_{n-1}\right)^{2} \leq \lambda_{1} \delta_{1}^{2}+\cdots+\lambda_{n-1} \delta_{n-1}^{2}-\epsilon \kappa
$$

where we have defined $\delta_{k}=(1-\epsilon) g_{j, k} / 2$ for each $k=1, \ldots, n-1$. If $\epsilon \in(0,1)$ is sufficiently small the right-hand side of $(24)$ is positive and so the region $\mathcal{E}_{j} \subset \mathcal{H}_{n-1}$ is an ellipsoid. Importantly $\mathcal{E}_{j}$ is bounded, closed and convex. We have the following general result.

Theorem 4 Let $\mathcal{S}_{n-1}=\left\{\boldsymbol{p} \in \mathbb{R}^{n} \mid \boldsymbol{p} \geq \mathbf{0}\right.$ and $\left.\mathbf{1}^{T} \boldsymbol{p}=1\right\}$ and let $W=\left[w_{i j}\right] \in \mathbb{R}^{n \times n}$ be symmetric and positive definite with $w_{i j} \geq 0$. Assume that $W^{-1} \mathbf{1}>0$. Choose $\epsilon \in(0,1)$ and fix $j \in\{1, \ldots, n\}$. Define $\mathcal{E}_{j}=\mathcal{E}_{j, \epsilon}$ by

$$
\mathcal{E}_{j}=\left\{\boldsymbol{p} \in \mathcal{H}_{n-1} \mid(1-\epsilon) \boldsymbol{e}_{j}^{T} W \boldsymbol{p} \geq \boldsymbol{p}^{T} W \boldsymbol{p}\right\}
$$

and let $\mathcal{D}_{j}=\mathcal{D}_{j, \epsilon}$ be defined by $\mathcal{D}_{j}=\mathcal{S}_{n-1} \cap \mathcal{E}_{j}$. Let $\boldsymbol{p}(1)=W^{-1} \mathbf{1} /\left(\mathbf{1}^{T} W^{-1} \mathbf{1}\right)$ and define

$$
\boldsymbol{q}(t)=\frac{\boldsymbol{p}(t) \circ[W \boldsymbol{p}(t)]}{\boldsymbol{p}(t)^{T} W \boldsymbol{p}(t)} \quad \text { and } \quad \boldsymbol{p}(t+1)=(1-\epsilon) \boldsymbol{q}(t)+\epsilon \boldsymbol{r}(t)
$$

for each $t \in \mathbb{N}$ where $\{\boldsymbol{r}(t)\}_{t \in \mathbb{N}} \in \mathcal{S}_{n-1}^{\infty}$ is an arbitrarily chosen sequence of vectors. If $\boldsymbol{p}(s) \in \mathcal{D}_{j}$ for some $s \in \mathbb{N}$ then $p_{j}(s+1) \geq p_{j}(s)$. That is, if the population distribution vector $\boldsymbol{p}$ lies in the proliferation set $\mathcal{D}_{j, \epsilon}$ at generation $s$, then the relative frequency $p_{j}$ of genotype $\mathcal{G}_{j}$ will increase in the transition to generation $s+1$ irrespective of the perturbation. If the vertex $\boldsymbol{e}_{j}$ is a stable fixed point in the unperturbed case and $\epsilon \in[0,1)$ is sufficiently small then we can find $\alpha_{j, \epsilon} \in(0,1)$ such that $\mathcal{C}_{j, \epsilon}=\left\{\boldsymbol{p} \in \mathcal{S}_{n-1} \mid p_{j} \geq \alpha_{j, \epsilon}\right\}$ is a capture region for genotype $\mathcal{G}_{j}$. 
Proof Since $\boldsymbol{p}(s) \in \mathcal{D}_{j}=\mathcal{S}_{n-1} \cap \mathcal{E}_{j}$ we have $\boldsymbol{p}(s) \in \mathcal{E}_{j}$ and hence it follows from the definition of $\mathcal{E}_{j}$ that $p_{j}(s+1) \geq p_{j}(s)$.

Now suppose $\boldsymbol{e}_{j}$ is a stable fixed point for the unperturbed evolution. When $\epsilon=0$ we have $\boldsymbol{e}_{j} \in \mathcal{E}_{j, 0}$ and $\boldsymbol{p}^{*}=W^{-1} \mathbf{1} /\left(\mathbf{1}^{T} W^{-1} \mathbf{1}\right) \in \mathcal{E}_{j, 0}$. Since $\boldsymbol{e}_{j}$ is a stable fixed point there exists some $\alpha_{j, 0}>0$ such that $\boldsymbol{v}_{i, j}(\alpha)=\alpha \boldsymbol{e}_{j}+(1-\alpha) \boldsymbol{e}_{i} \in \mathcal{E}_{j, 0}$ for all $i \neq j$ and all $\alpha \geq \alpha_{j, 0}$. Since $\mathcal{E}_{j, 0} \subset \mathcal{H}_{n-1}$ is an ellipsoid it follows that $\boldsymbol{v}_{i, j}(\alpha) \in \mathcal{E}_{j, 0}$ for all $\alpha \in\left[\alpha_{j, 0}, 1\right]$ and all $i \neq j$. Let $\mathcal{P}_{j}(\alpha)$ be the polyhedral region of dimension $n-2$ defined by

$$
\mathcal{P}_{j}(\alpha)=\left\{\boldsymbol{p} \in \mathcal{S}_{n-1} \mid p_{j}=\alpha\right\}
$$

for each $\alpha \in[0,1]$. The set $\mathcal{P}_{j}(\alpha)$ is closed and convex with vertices $\boldsymbol{v}_{i, j}(\alpha)=\alpha \boldsymbol{e}_{j}+(1-\alpha) \boldsymbol{e}_{i}$ for each $i \in\{1,2, \ldots, n\}$ with $i \neq j$. Since $\boldsymbol{v}_{i, j}\left(\alpha_{j, 0}\right) \in \mathcal{D}_{j}$ for each $i \neq j$ it follows that $\mathcal{P}_{j}\left(\alpha_{j, 0}\right) \subset \mathcal{D}_{j}$.

Now suppose $\alpha_{j} \in\left(\alpha_{j, 0}, 1\right)$. The closed convex polyhedral region $\mathcal{P}_{j}\left(\alpha_{j}\right) \subset \mathcal{S}_{n-1}$ is defined by the vertices $\boldsymbol{v}_{i, j}\left(\alpha_{j}\right) \in \operatorname{int}\left(\mathcal{E}_{j, 0}\right)$ for all $i \neq j$ and so we also have $\mathcal{P}_{j}\left(\alpha_{j}\right) \subset \operatorname{int}\left(\mathcal{E}_{j, 0}\right)$. Continuity with respect to $\epsilon$ for the set $\mathcal{E}_{j, \epsilon}$ means that for some $\epsilon \in(0,1)$ with $\epsilon$ sufficiently close to zero we must have $\mathcal{P}_{j}\left(\alpha_{j}\right) \subset \operatorname{int}\left(\mathcal{E}_{j, \epsilon}\right)$. Therefore we also have $\mathcal{P}_{j}\left(\alpha_{j}\right) \subset \mathcal{D}_{j, \epsilon}$. For each such $\epsilon$ we can define $\alpha_{j, \epsilon}$ as the maximum possible value for $\alpha_{j}$ such that $\mathcal{P}_{j}\left(\alpha_{j}\right) \subset \mathcal{D}_{j, \epsilon}$. The associated polyhedral set $\mathcal{P}_{j, \epsilon}$ is a barrier set that isolates vertex $V_{j}$ from the other vertices of the simplex $\mathcal{S}_{n-1}$. Now we define $\mathcal{C}_{j, \epsilon}=\left\{\boldsymbol{p} \in \mathcal{S}_{n-1} \mid p_{j} \geq \alpha_{j, \epsilon}\right\}$ where $p_{j}=\boldsymbol{e}_{j}^{T} \boldsymbol{p}$. We wish to show that $\mathcal{C}_{j, \epsilon}$ is a capture region for genotype $\mathcal{G}_{j}$.

Define $\boldsymbol{v}(\alpha)=\alpha \boldsymbol{e}_{j}+(1-\alpha) \boldsymbol{u}$ for some $\boldsymbol{u} \in \mathcal{S}_{n-1}$ with $u_{j}=\boldsymbol{e}_{j}{ }^{T} \boldsymbol{u}=0$ and all $\alpha \in[0,1]$. Since $v_{j}(\alpha)=\boldsymbol{e}_{j}{ }^{T} \boldsymbol{v}(\alpha)=\alpha$ it follows that $\boldsymbol{v}(\alpha) \in \mathcal{P}_{j}(\alpha)$. Define

$$
\begin{aligned}
\varphi(\alpha) & =\frac{v_{j}(\alpha)[W \boldsymbol{v}(\alpha)]_{j}}{\boldsymbol{v}(\alpha)^{T} W \boldsymbol{v}(\alpha)} \\
& =\frac{w_{j j} \alpha^{2}+\left(\boldsymbol{e}_{j}^{T} W \boldsymbol{u}\right) \alpha(1-\alpha)}{\left(w_{j j}-2 \boldsymbol{e}_{j}^{T} W \boldsymbol{u}+\boldsymbol{u}^{T} W \boldsymbol{u}\right) \alpha^{2}+2\left(\boldsymbol{e}_{j}^{T} W \boldsymbol{u}-\boldsymbol{u}^{T} W \boldsymbol{u}\right) \alpha+\boldsymbol{u}^{T} W \boldsymbol{u}} \\
& =\frac{f(\alpha)}{g(\alpha)}
\end{aligned}
$$

for all $\alpha \in[0,1]$. We wish to show that $\varphi^{\prime}(\alpha)>0$ for all $\alpha \in(0,1)$. We have $\varphi^{\prime}(\alpha)=0 \Longleftrightarrow$ $f^{\prime}(\alpha) g(\alpha)-f(\alpha) g^{\prime}(\alpha)=0$ and some elementary algebra shows that this latter condition is equivalent to

$$
\begin{aligned}
& {\left[w_{j j}\left(\boldsymbol{e}_{j}^{T} W \boldsymbol{u}\right)-2 w_{j j}\left(\boldsymbol{u}^{T} W \boldsymbol{u}\right)+\left(\boldsymbol{e}_{j}^{T} W \boldsymbol{u}\right)\left(\boldsymbol{u}^{T} W \boldsymbol{u}\right)\right] \alpha^{2}} \\
& \quad+2\left[w_{j j}\left(\boldsymbol{u}^{T} W \boldsymbol{u}\right)-\left(\boldsymbol{e}_{j}^{T} W \boldsymbol{u}\right)\left(\boldsymbol{u}^{T} W \boldsymbol{u}\right)\right] \alpha+\left(\boldsymbol{e}_{j}^{T} W \boldsymbol{u}\right)\left(\boldsymbol{u}^{T} W \boldsymbol{u}\right)=0 .
\end{aligned}
$$

The condition (26) can be rewritten in the form

$$
2 w_{j j}\left(\boldsymbol{u}^{T} W \boldsymbol{u}\right) \alpha(1-\alpha)+\left(\boldsymbol{e}_{j}^{T} W \boldsymbol{u}\right)\left(\boldsymbol{u}^{T} W \boldsymbol{u}\right)(1-\alpha)^{2}+\left(\boldsymbol{e}_{j}^{T} W \boldsymbol{u}\right) w_{j j} \alpha^{2}=0
$$


and since $w_{j j}=\boldsymbol{e}_{j}^{T} W \boldsymbol{e}_{j}>0, \boldsymbol{e}_{j}{ }^{T} W \boldsymbol{u} \geq 0$ and $\boldsymbol{u}^{T} W \boldsymbol{u}>0$ the left-hand side is positive for all $\alpha \in(0,1)$. Thus equality is not possible and hence $\varphi^{\prime}(\alpha) \neq 0$ for all $\alpha \in(0,1)$. Since $\varphi(0)=0$ and $\varphi(1)=1$ it follows that $\varphi^{\prime}(\alpha)>0$ for all $\alpha \in(0,1)$.

Now suppose $\boldsymbol{p}(s) \in \mathcal{C}_{j, \epsilon}$ at some time $s$. Write $\boldsymbol{p}(s)=\boldsymbol{v}(\alpha)=\alpha \boldsymbol{e}_{j}+(1-\alpha) \boldsymbol{u}$ for some $\alpha \geq \alpha_{j, \epsilon}$ and some $\boldsymbol{u} \in \mathcal{S}_{n-1}$ with $u_{j}=\boldsymbol{e}_{j}{ }^{T} \boldsymbol{u}=0$. It follows that

$$
\begin{aligned}
(1-\epsilon) q_{j}(s) & =(1-\epsilon) \frac{v_{j}(\alpha)[W \boldsymbol{v}(\alpha)]_{j}}{\boldsymbol{v}(\alpha)^{T} W \boldsymbol{v}(\alpha)} \\
& =(1-\epsilon) \varphi(\alpha) \\
& \geq(1-\epsilon) \varphi\left(\alpha_{j, \epsilon}\right) \\
& =(1-\epsilon) \frac{v_{j}\left(\alpha_{j, \epsilon}\right)\left[W \boldsymbol{v}\left(\alpha_{j, \epsilon}\right)\right]_{j}}{\boldsymbol{v}\left(\alpha_{j, \epsilon}\right)^{T} W \boldsymbol{v}\left(\alpha_{j, \epsilon}\right)} \\
& \geq \alpha_{j, \epsilon}
\end{aligned}
$$

because $\boldsymbol{v}\left(\alpha_{j, \epsilon}\right) \in \mathcal{P}_{j}\left(\alpha_{j, \epsilon}\right) \subset \mathcal{D}_{j, \epsilon}$. Therefore $p_{j}(s+1)=(1-\epsilon) q_{j}(s) \geq \alpha_{j, \epsilon}$ and hence $\boldsymbol{p}(s+1) \in \mathcal{C}_{j, \epsilon}$.

Theorem 5 Let $\mathcal{S}_{n-1}=\left\{\boldsymbol{p} \in \mathbb{R}^{n} \mid \boldsymbol{p} \geq \mathbf{0}\right.$ and $\left.\mathbf{1}^{T} \boldsymbol{p}=1\right\}$. Let $W=\left[w_{i j}\right] \in \mathbb{R}^{n \times n}$ be symmetric and positive definite with $w_{i j} \geq 0$ and assume that $W^{-1} \mathbf{1}>0$. If the stable vertices in the unperturbed evolution are denoted by $\boldsymbol{e}_{j(s)}$ for each $s=1, \ldots, \ell$ and $\epsilon \in[0,1)$ is sufficiently small then we can find $\alpha_{j(s), \epsilon} \in(0,1)$ such that $\mathcal{C}_{j(s), \epsilon}=\left\{\boldsymbol{p} \in \mathcal{S}_{n-1} \mid p_{j(s)} \geq \alpha_{j(s), \epsilon}\right\}$ is a capture region for genotype $\mathcal{G}_{j(s)}$ for each $s=1, \ldots, \ell$. If $\boldsymbol{r}=\{\boldsymbol{r}(t)\}_{t \in \mathbb{N}} \in \mathcal{S}_{n-1}$ is an outcome generated by a sequence of independent identically distributed random vectors with a constant probability $\mathbb{P}\left[\boldsymbol{r}(t) \in \mathcal{S}_{n-1} \mid r_{j(s)}(t)>\kappa\right]=\tau_{s}(\kappa)>0$ for each $t \in \mathbb{N}$ and each $s=1, \ldots, \ell$ when $\kappa \in(0,1)$ then $\boldsymbol{p}(t)$ is eventually captured in one or other of the capture regions with probability one.

Proof For each capture region $\mathcal{C}_{j(s), \epsilon}$ we define an open neighbourhood $N\left(\mathcal{C}_{j(s), \epsilon}, \delta_{s}\right) \supseteq \mathcal{C}_{j(s), \epsilon}$ as the set of points $\boldsymbol{p} \in \mathcal{S}_{n-1}$ with $\|\boldsymbol{p}-\boldsymbol{q}\|<\delta_{s}$ for some $\boldsymbol{q} \in \mathcal{C}_{j(s), \epsilon}$. Choose $\delta_{s}>0$ sufficiently small to ensure that if $\boldsymbol{p}(t) \in N\left(\mathcal{C}_{j(s), \epsilon}, \delta_{s}\right)$ then, in the unperturbed evolution, we must have $p(t+1) \in \mathcal{C}_{j(s), \epsilon}$. Let $\delta=\min _{s} \delta_{s}$ over all $s \in\{1, \ldots, \ell\}$. Clearly $\delta>0$. Consequently for the perturbed evolution, if $\boldsymbol{p}(t) \in N\left(\mathcal{C}_{j(s), \epsilon}, \delta\right)$, there must be a positive probability $\gamma_{s}$ that $p(t+1) \in \mathcal{C}_{j(s), \epsilon}$. Now define a compact set

$$
K_{\epsilon}=\mathcal{S}_{n-1} \backslash \bigcup_{s=1}^{\ell} N\left(\mathcal{C}_{j(s), \epsilon}, \delta / 2\right) .
$$

For each $\boldsymbol{p} \in K_{\epsilon}$ and each $s \in\{1, \ldots, \ell\}$ there is a value $\pi(\boldsymbol{p}, s) \in(0,1)$ such that if $\boldsymbol{p}(1)=\boldsymbol{p}$ and $\boldsymbol{r}_{j(s)}(t)>\pi(\boldsymbol{p}, s)$ for all $t \in \mathbb{N}$ then there is a finite number $n(\boldsymbol{p}, s) \in \mathbb{N}$ such that $\boldsymbol{x}(t) \in N\left(\mathcal{C}_{j(s), \epsilon}, \delta / 2\right)$ for all $t \geq n(\boldsymbol{p}, s)$. By continuity of the mapping there must be an open neighbourhood $N(\boldsymbol{p}, \varepsilon(\boldsymbol{p}))$ with $\varepsilon(\boldsymbol{p})>0$ such that for all sequences with $\boldsymbol{r}_{j(s)}(t)>\pi(\boldsymbol{p}, s)$ for all $t \in \mathbb{N}$ and $\boldsymbol{p}(1)=\boldsymbol{x} \in$ $N(\boldsymbol{p}, \varepsilon(\boldsymbol{p}))$ we will have $\boldsymbol{p}(t) \in N\left(\mathcal{C}_{j(s), \epsilon}, \delta\right)$ for all $t \geq n(\boldsymbol{p}, s)$. Now we have

$$
K_{\epsilon} \subseteq \bigcup_{\boldsymbol{p} \in K_{\epsilon}} N(\boldsymbol{p}, \varepsilon(\boldsymbol{p}))
$$


and we can apply the Heine-Borel theorem to deduce that there is some finite sub-collection $\left\{N\left(\boldsymbol{p}_{r}, \varepsilon_{r}\right)\right\}_{r=1}^{m}$, where we have written $\varepsilon_{r}$ in place of $\varepsilon\left(\boldsymbol{p}_{r}\right)$, which also covers $K_{\epsilon}$. Let $\gamma=\min _{s} \gamma_{s}, n=\max _{r, s} n\left(\boldsymbol{p}_{r}, s\right)$, and $\pi=\max _{r, s} \pi\left(\boldsymbol{p}_{r}, s\right) \in(0,1)$ over all $r=1, \ldots, m$ and $s=1, \ldots, \ell$. If $\boldsymbol{p}(1) \in K_{\epsilon}$ there is a positive probability $\pi \cdot \gamma$ that

$$
\boldsymbol{p}(n+1) \in \bigcup_{s=1}^{\ell} \mathcal{C}_{j(s), \epsilon} .
$$

If $\boldsymbol{p}(n+1) \in K_{\epsilon}$ then the above argument can be repeated. The chance that $\boldsymbol{p}(t)$ will be captured after $q$ such episodes is at least equal to

$$
\pi \gamma\left[1+(1-\pi \gamma)+(1-\pi \gamma)^{2}+\cdots+(1-\pi \gamma)^{q-1}\right]=1-(1-\pi \gamma)^{q} \rightarrow 1
$$

as $q \rightarrow \infty$. Thus capture in one of the regions $\mathcal{C}_{j(s), \epsilon}$ is inevitable.

Remark 6 Note that the region $\mathcal{E}_{j, \epsilon}$ for $\epsilon \in[0,1]$ is not necessarily a capture region. Suppose there are two conditionally dominant genotypes $\mathcal{G}_{j}$ and $\mathcal{G}_{k}$ and that $\boldsymbol{p}_{\{1, \ldots, n\}}^{*}=W^{-1} \mathbf{1} /\left(\mathbf{1}^{T} W^{-1} \mathbf{1}\right) \in$ $\operatorname{int}\left(\mathcal{S}_{n-1}\right)$ is an unstable fixed point. There will be stable fixed points at $\boldsymbol{e}_{j}$ and $\boldsymbol{e}_{k}$ and there will also be an unstable fixed point $\boldsymbol{p}_{\{j, k\}}^{*}$ on the one-dimensional face $\mathcal{F}_{\{j, k\}}$. Since $\boldsymbol{e}_{j}, \boldsymbol{p}_{\{1, \ldots, n\}}^{*}, \boldsymbol{p}_{\{j, k\}}^{*} \in \mathcal{E}_{j, 0}$ and $\boldsymbol{e}_{k}, \boldsymbol{p}_{\{1, \ldots, n\}}^{*}, \boldsymbol{p}_{\{j, k\}}^{*} \in \mathcal{E}_{k, 0}$ and because both regions are ellipsoids it follows that $\operatorname{int}\left(\mathcal{E}_{j, 0} \cap \mathcal{E}_{k, 0}\right) \neq$ $\emptyset$. Thus there are points in this region where both $p_{j}$ and $p_{k}$ will increase during the unperturbed evolution. Because the regions are deformed continuously with respect to $\epsilon$ it also follows that we will have $\operatorname{int}\left(\mathcal{E}_{j, \epsilon} \cap \mathcal{E}_{k, \epsilon}\right) \neq \emptyset$ for some sufficiently small $\epsilon \in(0,1)$. Thus there are also points where both $p_{j}$ and $p_{k}$ will increase during the perturbed evolution.

Remark 7 The fact that Theorem 2 holds for all $W=\left[w_{i j}\right] \in \mathbb{R}^{n \times n}$ with $w_{i j}=w_{j i} \geq 0$ raises an interesting question about Theorems 4 and 5, where the results are established using a convex capture set that lies within a key convex elliptical set. The definition of the elliptical set depends on the assumption that $W$ is positive definite. When $W$ is indefinite the elliptical set is replaced by a hyperbolic set that is no longer convex.

\section{Extension to system matrices that are not symmetric}

Let $W=\left[w_{i j}\right] \in \mathbb{R}^{n \times n}$ with $w_{i j} \geq 0$ for all $i, j \in\{1, \ldots, n\}$ and suppose that $W$ is not symmetric. Although it is more usual to define quadratic forms using a symmetric matrix we nevertheless say that $W$ is positive definite if $\boldsymbol{x}^{T} W \boldsymbol{x}>0$ for all $\boldsymbol{x} \in \mathbb{R}^{n}$ with $\boldsymbol{x} \neq \mathbf{0}$. Since $\boldsymbol{x}^{T} W^{T} \boldsymbol{x}=\left(\boldsymbol{x}^{T} W \boldsymbol{x}\right)^{T}=$ $\boldsymbol{x}^{T} W \boldsymbol{x}$ it follows that if we define the symmetric matrix $S=\left(W+W^{T}\right) / 2$ then $\boldsymbol{x}^{T} S \boldsymbol{x}=\boldsymbol{x}^{T} W \boldsymbol{x}$. Now consider the perturbed replicator equation with system matrix $W$ and perturbation parameter $\epsilon \in(0,1)$. Define the set $\mathcal{E}_{j}=\mathcal{E}_{j, \epsilon}$ by setting

$$
\mathcal{E}_{j}=\left\{\boldsymbol{p} \in \mathcal{H}_{n-1} \mid(1-\epsilon) \boldsymbol{e}_{j}{ }^{T} W \boldsymbol{p} \geq \boldsymbol{p}^{T} W \boldsymbol{p}\right\}=\left\{\boldsymbol{p} \in \mathcal{H}_{n-1} \mid(1-\epsilon) \boldsymbol{e}_{j}{ }^{T} W \boldsymbol{p} \geq \boldsymbol{p}^{T} S \boldsymbol{p}\right\}
$$


where $\mathcal{H}_{n-1} \subset \mathbb{R}^{n}$ is the hyperplane defined by $\boldsymbol{p}^{T} \mathbf{1}=1$ for all $\boldsymbol{p} \in \mathbb{R}^{n}$. Write $\boldsymbol{p}=\boldsymbol{p}^{*}+\boldsymbol{u}$ and $\boldsymbol{e}_{j}=\boldsymbol{p}^{*}+\boldsymbol{f}_{j}$ where $\boldsymbol{p}^{*}=S^{-1} \mathbf{1} /\left(\mathbf{1}^{T} S^{-1} \mathbf{1}\right) \in \mathcal{H}_{n-1}$. Now we can write $(27)$ in the form

$$
\begin{aligned}
(1-\epsilon)\left(\boldsymbol{p}^{*}+\boldsymbol{f}_{j}\right)^{T} W\left(\boldsymbol{p}^{*}+\boldsymbol{u}\right) \geq\left(\boldsymbol{p}^{*}+\boldsymbol{u}\right)^{T} S\left(\boldsymbol{p}^{*}+\boldsymbol{u}\right) \\
\quad \Longleftrightarrow \quad-\epsilon \kappa+(1-\epsilon) \mu_{j}+(1-\epsilon)\left(\boldsymbol{p}^{*}+\boldsymbol{f}_{j}\right)^{T} W \boldsymbol{u} \geq \boldsymbol{u}^{T} S \boldsymbol{u} \\
\quad \Longleftrightarrow \quad-\epsilon \kappa+(1-\epsilon) \mu_{j}+(1-\epsilon) \boldsymbol{e}_{j}^{T} W \boldsymbol{u} \geq \boldsymbol{u}^{T} S \boldsymbol{u}
\end{aligned}
$$

where $\kappa=\boldsymbol{p}^{* T} W \boldsymbol{p}^{*}=\boldsymbol{p}^{* T} S \boldsymbol{p}^{*}=1 /\left(\mathbf{1}^{T} S^{-1} \mathbf{1}\right)>0$ and $\mu_{j}=\boldsymbol{f}_{j}^{T} W \boldsymbol{p}^{*}$ and where we have used $\boldsymbol{u}^{T} S \boldsymbol{p}^{*}=\boldsymbol{u}^{T} \mathbf{1} /\left(\mathbf{1}^{T} S^{-1} \mathbf{1}\right)=0$ in much the same way as we did in Section 6.2. If we define the orthogonal projection matrix $P=I-\mathbf{1 1}^{T} / n$ then (28) can be rewritten as

$$
-\epsilon \kappa+(1-\epsilon) \mu_{j}+(1-\epsilon) \boldsymbol{e}_{j}^{T} W \boldsymbol{u} \geq \boldsymbol{u}^{T} X \boldsymbol{u}
$$

where we have defined $X=P^{T} S P$. Since $X \boldsymbol{u}=S \boldsymbol{u}$ when $\boldsymbol{u} \in \mathcal{S}_{n-1}$ but $X \mathbf{1}=\mathbf{0}$ it follows that $X$ is positive semi-definite with eigenvalues $\lambda_{1} \geq \cdots \geq \lambda_{n-1}>\lambda_{n}=0$ and there exists an orthogonal matrix $Q$ such that $Q^{T} X Q=\Lambda$ where $\Lambda=\operatorname{diag}\left(\lambda_{j}\right)$ is the diagonal matrix of eigenvalues. Therefore

$$
\begin{aligned}
& -\epsilon \kappa+(1-\epsilon) \mu_{j}+(1-\epsilon) \boldsymbol{e}_{j}^{T} W \boldsymbol{u} \geq \boldsymbol{u}^{T} S \boldsymbol{u} \\
& \quad \Longleftrightarrow \quad-\epsilon \kappa+(1-\epsilon) \mu_{j}+(1-\epsilon) \boldsymbol{e}_{j}^{T}(W Q)\left(Q^{T} \boldsymbol{u}\right) \geq\left(Q^{T} \boldsymbol{u}\right)^{T}\left(Q^{T} X Q\right)\left(Q^{T} \boldsymbol{u}\right) \\
& \quad \Longleftrightarrow \quad-\epsilon \kappa+(1-\epsilon) \mu_{j}+(1-\epsilon) \boldsymbol{g}_{j}^{T} \boldsymbol{v} \geq \boldsymbol{v}^{T} \Lambda \boldsymbol{v}
\end{aligned}
$$

where $\boldsymbol{g}_{j}=Q^{T} W^{T} \boldsymbol{e}_{j}$ and $\boldsymbol{v}=Q^{T} \boldsymbol{u}$. Now (30) can be rewritten as

$$
\lambda_{1} v_{1}^{2}+\cdots+\lambda_{n-1} v_{n-1}^{2} \leq(1-\epsilon)\left[g_{j, 1} v_{1}+\cdots+g_{j, n-1} v_{n-1}\right]+(1-\epsilon) \mu_{j}-\epsilon \kappa
$$

or equivalently as

$$
\lambda_{1}\left(v_{1}-\delta_{1}\right)^{2}+\cdots+\lambda_{n-1}\left(v_{n-1}-\delta_{n-1}\right)^{2} \leq \lambda_{1} \delta_{1}^{2}+\cdots+\lambda_{n-1} \delta_{n-1}^{2}+(1-\epsilon) \mu_{j}-\epsilon \kappa
$$

where we have defined $\delta_{k}=(1-\epsilon) g_{j, k} /\left(2 \lambda_{k}\right)$ for each $k=1, \ldots, n-1$. Therefore the region $\mathcal{E}_{j} \subset \mathcal{H}_{n-1}$ is an ellipsoid. If

$$
\left(g_{j, 1}^{2} / \lambda_{1}+\cdots+g_{j, n-1}^{2} / \lambda_{n-1}\right) / 4+\mu_{j}>0
$$

then the right-hand side of (31) is positive for sufficiently small $\epsilon \in[0,1)$. Hence there is some $\epsilon_{0}>0$ such that $\mathcal{E}_{j, \epsilon} \neq \emptyset$ for all $\epsilon \in\left[0, \epsilon_{0}\right)$. Theorem 4 can now be established for matrices that are not symmetric by following essentially the same arguments that we used earlier.

\section{Some remarks about the perturbation analysis}

The initial motivation for this study was our interest in singular perturbations where the behaviour of the perturbed system changes radically from the behaviour of the unperturbed system no matter 
how small the perturbation. However our work here shows that small perturbations in population distribution are always regular if the evolution is defined by the replicator equation with a positive definite system matrix. Indeed, for all conditionally dominant genotypes $\mathcal{G}_{j}$ and all sufficiently small $\epsilon \in(0,1)$, there is an associated capture set $\mathcal{C}_{j, \epsilon}$ and as $\epsilon \downarrow 0$ the set $\mathcal{C}_{j, \epsilon}$ converges to the corresponding capture set $\mathcal{C}_{j, 0}$ for the unperturbed system.

Nevertheless there are positive values of $\epsilon$ where the perturbation is singular. For each conditionally dominant genotype $\mathcal{G}_{j}$ and each sufficiently small $\epsilon \in[0,1)$ there is a non-trivial capture region

$$
\mathcal{C}_{j, \epsilon}=\left\{\boldsymbol{p}=\left[p_{i}\right] \in \mathcal{S}_{n-1} \mid p_{j} \in\left[\alpha_{j, \epsilon}, 1\right]\right\}
$$

with the property that $\boldsymbol{p}(s) \in \mathcal{C}_{j, \epsilon}$ implies $\boldsymbol{p}(t) \in \mathcal{C}_{j, \epsilon}$ for all $t>s$. Define

$$
\epsilon_{j, \max }=\sup \left\{\epsilon \in[0,1] \mid \alpha_{j, \epsilon}<1\right\}
$$

and let $\boldsymbol{p}(1)=\boldsymbol{p}^{*} \in \mathcal{S}_{n-1}$ be the point where the potential $w(\boldsymbol{p})$ is minimized. For each $\epsilon \in\left(0, \epsilon_{j, \max }\right)$ there is a positive probability $\mu_{j, \epsilon}>0$ that the perturbed evolution will be captured near $\boldsymbol{e}_{j}$ in the capture region $\mathcal{C}_{j, \epsilon}$. For $\epsilon \in\left(\epsilon_{j, \max }, 1\right]$ we have $\mathcal{C}_{j, \epsilon}=\emptyset$ and hence $\mu_{j, \epsilon}=0$. Thus the behaviour of the system changes radically at $\epsilon=\epsilon_{j \text {, max }}$ and consequently we say that the perturbation is singular at $\epsilon=\epsilon_{j, \max }$.

When we claim that the probability of capture near vertex $V_{j}$ is zero if $\mathcal{C}_{j, \epsilon}=\emptyset$ one could literally say we are stating the obvious. If the capture set is empty then there must be a zero probability that $\boldsymbol{p}(t)$ can lie in the capture set. Is it possible that $\boldsymbol{p}(t)$ will remain in the vicinity of vertex $V_{j}$ when the corresponding capture set is empty? If one interprets this statement to mean that in some sense $\boldsymbol{p}(t)$ will always remain near vertex $V_{j}$ then the proof of Theorem 5 shows that this cannot be true. When $\boldsymbol{p}(t)$ does not lie in a capture region there is always a finite probability that a sufficiently long sequence of suitable perturbations will push $\boldsymbol{p}(t)$ into the vicinity of another vertex $V_{k}$. If one asks about the existence of an ergodic measure, which would necessarily depend on $\boldsymbol{p}(1)$ and on $\epsilon$, then there are two possibilities that spring to mind. If there are non-empty capture sets, then Theorem 5 shows that the population will finish up in one of these sets with probability one. In that case the probability associated with any neighbourhood of a vertex with an empty capture set is necessarily zero. If all capture sets are empty then one would first need to decide whether or not an ergodic measure exists. Any answer to this problem is beyond the scope of the current paper.

\section{Future work}

In our future work we are interested in the possibility of singular perturbations when the system is not positive definite. We are indebted to Maria Kleshnina [13] for suggesting the following example. 
Example 5 Consider the discrete-time replicator equation with

$$
W=\left[\begin{array}{ccc}
2 & 3 & 4 / 3 \\
4 / 3 & 2 & 3 \\
3 & 4 / 3 & 2
\end{array}\right]
$$

The unperturbed evolutionary paths defined by $\boldsymbol{q}=\boldsymbol{p} \circ(W \boldsymbol{p}) /\left(\boldsymbol{p}^{T} W \boldsymbol{p}\right)$ are closed cyclic paths which enclose the invariant point

$$
\boldsymbol{p}_{\{1,2,3\}}^{*}=\left[\begin{array}{c}
1 / 3 \\
1 / 3 \\
1 / 3
\end{array}\right] \text {. }
$$

There is a Lyapunov function $L(\boldsymbol{p})=\boldsymbol{p}^{T} W \boldsymbol{p} /\left(p_{1} p_{2} p_{3}\right)$ for this evolution and after some horrendous algebra we can show that $L(\boldsymbol{q})=L(\boldsymbol{p})$ thus justifying our observation that the unperturbed evolution always proceeds along a closed cyclic path. Now let $\epsilon>0$ be sufficiently small and consider a perturbed deterministic evolution defined by

$$
\boldsymbol{p}(t+1)=(1-\epsilon) \boldsymbol{p}(t) \circ[W \boldsymbol{p}(t)] /\left(\boldsymbol{p}(t)^{T} W \boldsymbol{p}(t)\right)+\epsilon \boldsymbol{r}
$$

where $\boldsymbol{r} \in \mathcal{S}_{2}$ is a constant. The Brouwer fixed point theorem shows that there must be a fixed point $\boldsymbol{p}_{\boldsymbol{r}}^{*} \in \mathcal{S}_{2}$ for this evolution. Surprisingly our numerical experiments suggest that if $\epsilon \in(0,1)$ is sufficiently small then the population distribution converges to $\boldsymbol{p}_{\boldsymbol{r}}^{*}$. If $\boldsymbol{r}=\boldsymbol{e}_{1}$ and $\epsilon \in(0,1 / 8)$ some elementary algebra shows that the fixed point is given by

$$
\boldsymbol{p}_{\epsilon}^{*}=\left[\begin{array}{c}
1 / 3+(1 / 3) \epsilon \\
1 / 3-(8 / 3) \epsilon \\
1 / 3+(7 / 3) \epsilon
\end{array}\right] .
$$

Even more surprisingly our experiments suggest that approximate convergence occurs for the evolution with sufficiently small random perturbations and that the capture region is a neighbourhood of the fixed point $\boldsymbol{p}_{\{1,2,3\}}^{*}$ for the unperturbed map. Figure 11 shows the evolution for 5000 generations with $\epsilon=0.04$ and a fixed perturbation $\boldsymbol{r}=\boldsymbol{e}_{1}$ on the left and a typical realisation of the evolution for 5000 generations with $\epsilon=0.04$ and a random perturbation $\{\boldsymbol{r}(t)\} \in \mathcal{S}_{2}^{5000}$ on the right.

The numerical calculations for Example 5 show that a semi-stable system with cyclic trajectories in the unperturbed state may become stable when the evolution is subject to a fixed deterministic perturbation of sufficiently small magnitude. Moreover it seems that the population converges to a uniquely determined fixed point. When the evolution is subject to a random perturbation of sufficiently small magnitude the population is apparently captured in a small region containing the fixed point $\boldsymbol{p}_{\{1,2,3\}}^{*} \in \operatorname{int}\left(\mathcal{S}_{2}\right)$ for the unperturbed evolution. We conclude that the perturbation is singular at $\epsilon=0$. We would like to establish a theoretical basis for these observations. Although we have used only standard techniques of linear algebra and elementary notions of convexity to establish the main results in this paper, there is no doubt that our methods could be seen as a particular application of the more general theory of discrete-time set-valued dynamical systems. It may be useful in our future work to make more explicit use of this theory. 

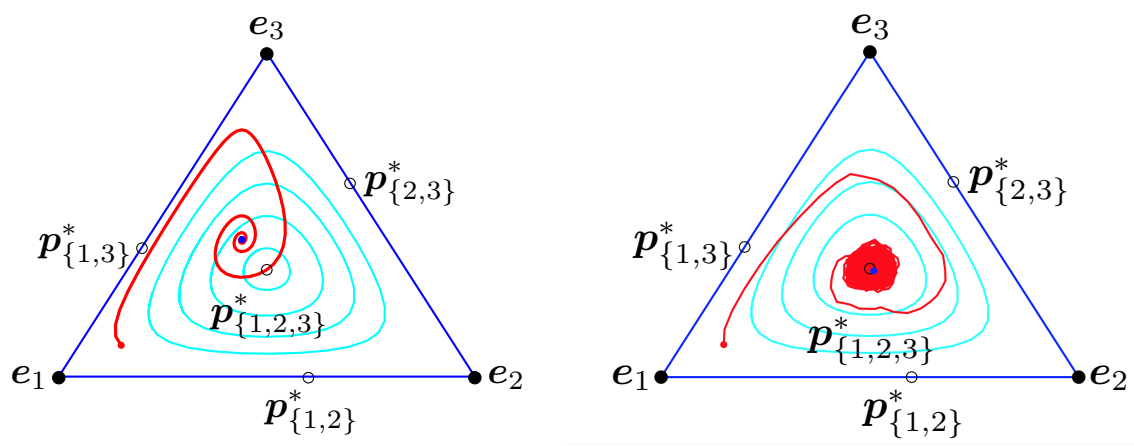

Figure 11: Evolutionary paths (thick red lines) in Example 5 for 5000 generations with $\epsilon=0.04$ and a fixed perturbation $\boldsymbol{r}=\boldsymbol{e}_{1}$ showing convergence to the fixed point $\boldsymbol{p}_{\epsilon}^{*}$ on the left and for 5000 generations of a typical realisation with $\epsilon=0.04$ and a random perturbation $\{\boldsymbol{r}(t)\} \in \mathcal{S}_{2}^{5000}$ showing approximate convergence to the fixed point $\boldsymbol{p}_{\{1,2,3\}}^{*}$ on the right. The cyclic curves (thin blue lines) for the unperturbed evolution with $\epsilon=0$ are also shown.

As mentioned in Section 1, there is an existing discrepancy in the perturbed behaviour established here, in the discrete-time evolution, and the perturbed behaviour established in $[1,2,6,7,9,10$, $11,15]$ for the continuous-time evolution. It would be interesting to see if an appropriate limiting argument applied to our discrete-time model would preserve the behaviour established here in a corresponding continuous-time model.

\section{Acknowledgements}

This work was supported by ARC Discovery Grant DP160101236. In particular this work was initiated when Geetika Verma visited Konstantin Avrachenkov at INRIA. We would like to thank our home institutions, UniSA and INRIA, for their support, and thank our colleagues Jerzy Filar and Maria Kleshnina for their input to our discussions about the dynamic behaviour of evolutionary systems. We also thank Gary Froyland for some timely advice about set-valued dynamical systems and two anonymous referees for their helpful suggestions.

\section{References}

[1] K. Avrachenkov and V.S. Borkar (2019), Metastability in stochastic replicator dynamics, Dynamic Games and Applications, 9, (2), 366-390.

[2] A. Cabrales (2000), Stochastic replicator dynamics, International Economic Review, 41, (2), 451-481. 
[3] Russell W. Cooper (1999). Coordination Games: Complementarities and Macroeconomics, Cambridge University Press. https://econpapers.repec.org/RePEc:cup:cbooks:9780521570176.

[4] Ross Cressman and Yi Tao (2014), The replicator equation and other game dynamics, Proc. Nat. Acad. Sc. (PNAS), 111, (3), 10810-10817. https://doi.org/10.1073/pnas.1400823111.

[5] R.A. Fisher (1930), The genetical theory of natural selection, Oxford, Clarendon Press. MR 2002c:92013.

[6] D. Foster and P. Young (1990), Stochastic evolutionary game dynamics, Theoretical population biology, 38, 219-232, [Corrigendum: Theoretical population biology, (1997), 51, 77-78].

[7] D. Fudenberg and C. Harris (1992), Evolutionary dynamics with aggregate shocks. Journal of Economic Theory, 57, (2), 420-441.

[8] Josef Hofbauer and Karl Sigmund (2003), Evolutionary game dynamics, Bulletin (New Series) American Mathematical Society, 40, (4), 479-519. S 0273-0979(03)00988-1.

[9] J. Hofbauer and L.A. Imhof (2009), Time averages, recurrence and transience in the stochastic replicator dynamics, Ann. Appl. Probab., 19, (4), 1347-1368.

[10] L.A. Imhof (2005), The long-run behavior of the stochastic replicator dynamics, Ann. Appl. Probab., 15, (1B), 1019-1045.

[11] R. Khasminskii and N. Potsepun (2006), On the replicator dynamics behavior under Stratonovich type random perturbations. Stochastics and Dynamics, 6.02, 197-211.

[12] J.F.C. Kingman(1961), A mathematical problem in population genetics, Proc. Camb. Phil. Soc. 57, 574-582.

[13] Maria Kleshnina (2018), Evolutionary games under incompetence $\&$ foraging strategies of marine bacteria, $\mathrm{PhD}$ thesis, University of Queensland.

[14] R. McGehee (1992), Attractors for Closed Relations on Compact Hausdorff Spaces, Indiana University Mathematics Journal, 41, 1165-1209.

[15] P. Mertikopoulos and Y. Viossat (2016), Imitation dynamics with payoff shocks. International Journal of Game Theory, 45, (1-2), 291-320.

[16] H. P. Mulholland and C. A. B. Smith (1959), An Inequality Arising in Genetical Theory, The American Mathematical Monthly, 66:8, 673-683. DOI: 10.1080/00029890.1959.11989387.

[17] W. H. Sandholm (2011), Population games and evolutionary dynamics, MIT Press, Cambridge.

[18] P. Schuster, K. Sigmund (1983) Replicator dynamics, J. Theor. Biology 100, 533-538. MR 85i:92006. 
[19] Peter D. Taylor and Leo B. Jonker (1978), Evolutionarily Stable Strategies, Mathematical Biosciences, 40, 145-156. MR 58:9351.

[20] J. Weibull (1995), Evolutionary game theory, MIT Press, Cambridge. 